\title{
Assessment of secondary bubble formation on a backward-facing step geometry
}

\author{
G. L. Juste, ${ }^{1, a)}$ P. Fajardo, ${ }^{2, b}$ and A. Guijarro ${ }^{3}$ \\ ${ }^{1}$ Aerospace Propulsion and Fluid Mechanics, Universidad Politécnica de Madrid, Plaza \\ Cardenal Cisneros 3, 28040 Madrid, Spain \\ ${ }^{2}$ Aerospace Engineering Group, Universidad Carlos III de Madrid, 28911 Leganés, Spain \\ ${ }^{3}$ Escuela Técnica Superior de Ingeniería Aeronáutica y del Espacio, Universidad Politécnica \\ de Madrid, Plaza Cardenal Cisneros 3, 28040 Madrid, Spain
}

(Received 16 January 2016; accepted 29 June 2016; published online 19 July 2016)

\begin{abstract}
Flow visualization experiments and numerical simulations were performed on a narrow three-dimensional backward-facing step (BFS) flow with the main objective of characterizing the secondary bubble appearing at the top wall. The BFS has been widely studied because of its geometrical simplicity as well as its ability to reproduce most of the flow features appearing in many applications in which separation occurs. A BFS test rig with an expansion ratio of 2 and two aspect ratios (AR $=4$ and $A R=8)$ was developed. Tests were performed at range of Reynolds numbers ranging from 50 to 1000; visualization experiments provided a qualitative description of secondary bubble and wall-jet flows. Large eddy simulations were carried out with two different codes for validation. Numerical solutions, once validated with experimental data from the literature, were used to acquire a deeper understanding of the experimental visualizations, to characterize the secondary bubble as a function of the flow variables (Reynolds and AR) and to analyze the effect of the secondary bubble on primary reattachment length. Finally, to decouple the sidewall effects due to the non-slip condition and the intrinsic flow three-dimensionality, numerical experiments with free-slip conditions over the sidewalls were computed. The main differences were as follows: When the non-slip condition is used, the secondary bubble appears at a Reynolds number of approximately 200, increases with the Reynolds number, and is limited to a small part of the span. This recirculation zone interacts with the wall-jets and causes the maximum and minimum lengths in the reattachment line of the primary recirculation. Under free slip conditions, the recirculation bubble appears at a higher Reynolds number and covers the entire channel span. Published by AIP Publishing. [http://dx.doi.org/10.1063/1.4958723]
\end{abstract}

\section{INTRODUCTION}

Flow separation, with a recirculation region and subsequent reattachment, is a classical problem in fluid mechanics. In internal flows, separation and reattachment caused by sudden changes in geometry occur in many engineering flows and around many engine components, such as cooling passages, turbine blades, rim seals, and combustion chambers. As a well-established fundamental case for benchmarking these flows, the backward-facing step (BFS) has received a lot of attention over the past decades because of its geometrical simplicity but complexity in terms of flow structure because many of the features encountered in other engineering separated flows are present.

The first numerical and experimental studies were focused on a two-dimensional geometry and were devoted to characterizing the flow structure, reattachment properties, and heat transfer. Those studies were conducted over a range of the main parameters of the problem, i.e., Reynolds

\footnotetext{
a) E-mail: gregorio.lopez@upm.es

b) E-mail: pablo.fajardo@uc3m.es
} 
number based on the height or on the hydraulic diameter of the channel, expansion ratio (duct height downstream the step to step size ratio), and inlet flow conditions (Eaton and Johnston ${ }^{1}$ and Lee and Mateescu ${ }^{2}$ ). In the experimental study of Armaly et al., ${ }^{3}$ a considerably large aspect ratio (channel width to height ratio upstream of the step) was selected for the channel to assume two-dimensionality of the flow at the channel middle plane.

In recent years, substantial effort has been dedicated to better understanding the three-dimensional aspects of this flow in the laminar, transitional, and turbulent regimes (Biswas et al. ${ }^{4}$ ). In addition to the characterization of the flow behavior, the study of three-dimensional BFS has been devoted to the understanding of two different phenomena: the three-dimensional stability of a flow over a backward-facing step and the effect of the sidewalls on the flow.

Detailed linear stability studies on the BFS have been reported by Kaiktsis et al., ${ }^{5}$ Barkley et al. ${ }^{6}$ Beaudoin et al. ${ }^{7}$ Blackburn et al.,${ }^{8}$ and Lanzerstorfer and Kuhlmann, ${ }^{9}$ among others. These works have reported results on the appearance of two and three-dimensional instabilities, convective and absolute critical Reynolds numbers, the wavelength of the instability, and the intrinsically unstable three-dimensional modes.

Nie and Armaly ${ }^{10-12}$ and Armaly et al. ${ }^{13}$ performed numerical simulations and experimental studies on three-dimensional BFS geometries with sidewalls. The flow was studied for various expansion ratios and Reynolds numbers on a model with an aspect ratio of $\mathrm{AR}=8$. Significant three-dimensional features were found, including the development of wall-jets or jet-like flow located downstream of the step near the sidewalls and pointing towards the channel mid-plane, a thinner upper wall recirculation zone modifying the two-dimensional configuration of the flow, and a spanwise distribution of the primary reattachment line, with a maximum reattachment length at the sidewall and a minimum reattachment length near the sidewall.

These sidewall induced three-dimensional features have been reported in other studies, such as those by Liou et al.,${ }^{14}$ Williams and Baker, ${ }^{15}$ Chiang and Sheu, ${ }^{16}$ Tylli et al.,${ }^{17}$ and Biswas et al. ${ }^{4}$ More recently, Malamataris ${ }^{18}$ performed a numerical simulation over a three-dimensional BFS with an aspect ratio $\mathrm{AR}=40$, an expansion ratio (ER) of 2, and Reynolds number ranging from 100 to 950. In that study, the Reynolds number was based on the mean velocity across the channel and the channel height at the outlet, $h+s=2 s$, where $s$ is the step height and $h$ is the inlet channel height. The study intended to provide greater insight into the effect of lateral walls on the flow. The two main results of this work were the distinction between wall effects and 3D (or end) effects and the influence of the lateral wall on the earlier onset of unsteadiness. The study was limited to a Reynolds number of $\mathrm{Re}=950$ because it was found to be the upper limit of a steady-state average solution for the authors' simulations.

All of the previously mentioned studies, except for the study by Armaly et al., ${ }^{13}$ were focused on geometries with large aspect ratios, in which three-dimensionality caused by lateral walls is essentially confined to the region next to the wall while the bulk flow remains unaffected, especially for low Reynolds numbers.

Iwai et al..$^{19}$ examined the effect of the channel aspect ratio at low Reynolds numbers concluding that at $\mathrm{Re}=250$, an $\mathrm{AR} \geq 16$ is required to achieve a two-dimensional region at the channel mid-plane.

Rani et $a l .^{20,21}$ and Sheu and Rani ${ }^{22}$ carried out a study on a geometry analogous to that used by Armaly et al., ${ }^{13}$ with $\mathrm{AR}=8$ and $\mathrm{ER}=2$, at laminar and transitional Reynolds numbers to provide insight into the flow behavior produced by the sidewalls and the shed vortical structures constrained behind the BFS. Analyses were performed via numerical transient simulations using a commercial Computational Fluid Dynamics (CFD) package (CFDRC). A detailed description of the flow behind the step was obtained, and the three-dimensionality of the flow was associated with Kelvin-Helmholtz instabilities and Taylor-Görtler-like vortices.

The effects of the inlet flow conditions (velocity profile and $\mathrm{Re}$ ), the expansion ratio of the channel, and the aspect ratio were studied experimentally by Tihon et al. ${ }^{23}$ The aspect ratio was varied between 16.7 and 41.7. Experiments were performed using electrodiffusion techniques to measure the wall shear rate along the experimental channel and two-dimensional numerical simulations with commercial CFD software (ANSYS-Fluent) were conducted to provide additional information about the global flow characteristics. The three-dimensionality of the temperature field in a 
thermoconvective flow was assessed using a combined experimental and numerical methodology by Juste and Fajardo. ${ }^{24}$

To the best of the authors' knowledge, few studies have addressed backward-facing step flow in narrow channels, i.e., at aspect ratios $\leq 8$. This lack of studies justifies the selection of lower aspect ratios in the analysis performed in the current manuscript. Uruba and Jonáś ${ }^{25}$ performed an experimental study on the recirculation zone appearing after the BFS in a narrow channel (aspect ratio $=4$ ), at relatively high Reynolds number on the order of $5 \times 10^{4}$. However, the actual geometries of internal flows systems used in heat transfer applications, such as turbine blade cooling channels with ribs, commonly have low aspect ratios. This gap in the literature demonstrated in the foregoing review motivated the current study.

This paper presents an experimental and numerical study, whose major objective was to provide deeper insight into the three-dimensionality occurring in the separated flow inside a narrow channel. In particular, this study examined the secondary recirculation bubble forming on the upper wall of the channel, its effect on the development of different three-dimensional flows, and the corresponding hydrodynamic action (intrinsic flow instability).

A dedicated experimental apparatus was built and instrumented. A backward-facing step channel model with an AR = 4 was mounted on a test rig. Smoke flow visualization techniques were used to observe secondary lateral recirculation bubble and its depth, as well as three-dimensional flow phenomena. The experiments were carried out for laminar flow at early transitional Reynolds numbers (based on the channel height) ranging from 100 to 1000. To assess the effect of the aspect ratio of the channel, additional visualizations were conducted using a model with an AR of 8 at two Reynolds numbers (300 and 500).

To gain deeper insight into the flow behavior, numerical simulations of the three-dimensional flow in the BFS geometry were performed. First, the numerical models were validated against experimental results. Different boundary conditions were then imposed on the lateral walls to understand the extrinsic effect of the sidewalls and differentiate it from the intrinsic effect of the three-dimensionality of the flow. Two different computational codes were used, and the results were compared with the experimental data. The final objective of the simulations was to assess the behavior of the secondary bubble and its effect on the flow behavior.

The paper is organized as follows. Section II provides a brief description of the experimental setup and the visualization tests performed. The section also presents preliminary results with a qualitative description of secondary bubble and wall-jet flows. Section III provides a description of the numerical models, boundary conditions, and the codes used for simulation. Moreover, in addition to the description of the computational models, the section discusses how the results were validated against experimental data, the match between two numerical codes is evaluated, and the experimental and numerical results are compared. In Section IV, numerical results are analyzed, focusing on the effect of the flow conditions on the secondary bubble, the effect of the secondary bubble on the primary eddy, and the effect of sidewalls on the flow. The effect of the sidewalls on the flow was assessed by imposing two different boundary conditions: slip vs. non-slip wall. Finally, the main results of this work are summarized in Section V.

\section{EXPERIMENTAL APPARATUS AND PRELIMINARY DESCRIPTION OF EXPERIMENTAL RESULTS}

\section{A. Description}

The BFS channel experimental apparatus used in this study is schematically depicted in Figure 1. Briefly, the rig consists of a polycarbonate channel with a sudden expansion (step), an appropriate air supply system, and a flow illumination and recording system for visualization purposes.

The airflow is supplied by a centrifugal blower with an adjustable rotational speed, thus allowing for mass flow rate control, measured by a hot-surface flow meter. A total pressure probe, connected to a pressure transducer, is placed downstream of the flow meter to record the temporal evolution and obtain the FFT. This measurement is intended to ensure that no disturbing frequencies 


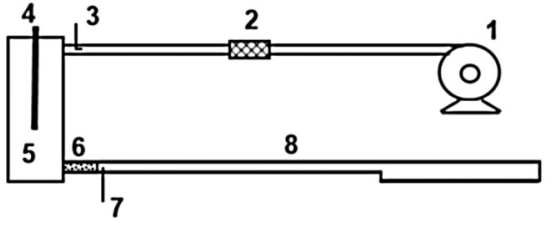

1. Centrifugal blower

2. Flowmeter

3. Pressure probe

4. Incense stick

5. Stagnation chamber

6. Honeycomb Straightener

7. Thermocouple

8. Channel

FIG. 1. Description of the experimental apparatus and optical setup.

alter the flow in the step. Additionally, a stagnation chamber is set upstream of the channel to minimize the residual pulses of the centrifugal blower and hence to achieve more homogenous flow at the inlet. Finally, the flow is passed through a honeycomb flow straightener before entering the channel to ensure flow uniformity at the channel inlet. The other side of the channel is opened to the free atmosphere.

The smoke used for flow visualization purposes was generated by burning incense sticks and introduced into the airflow at the stagnation chamber, where it mixed with the flow, yielding a heterogeneous but distributed mixture. A type-K thermocouple was set downstream of the stagnation chamber to evaluate the increase in temperature due to the smoke. This increment was determined to be smaller than the accuracy of the thermocouple $( \pm 1 \mathrm{~K})$.

Figure 2 shows a schematic drawing of the channel and optical setup. The test section was illuminated in planes (x-y and $\mathrm{x}-\mathrm{z}$ ) parallel to the flow stream direction by a light sheet generated by a continuous diode laser. The laser was placed in a micrometer-driven two-axis translation system with a travel range equal to the channel width, which allowed for different planes to be explored

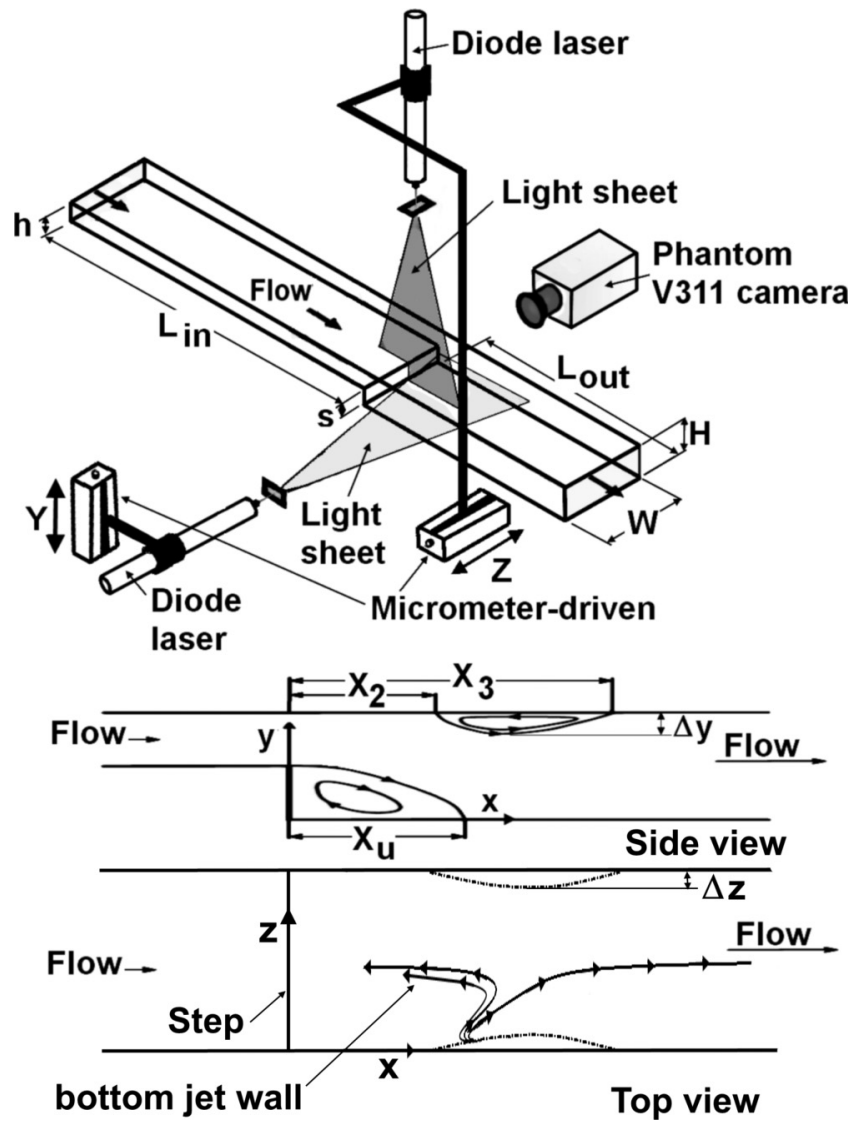

FIG. 2. (Top) Schematic drawing of the optical setup placed in the AR $=4$ channel. (Bottom) Lateral and top view schematic drawing of the main dimensions of the problem. 
TABLE I. Main geometrical parameters of the experimental arrangements.

\begin{tabular}{lcc}
\hline \hline & $\mathrm{AR}=4$ channel $(\mathrm{m})$ & $\mathrm{AR}=8$ channel $(\mathrm{m})$ \\
\hline Step size (s) & 0.006 & 0.006 \\
Channel height at inlet $(\mathrm{h})$ & 0.006 & 0.006 \\
Channel height $(\mathrm{H}=\mathrm{h}+\mathrm{s})$ & 0.012 & 0.012 \\
Channel width $(\mathrm{W})$ & 0.024 & 0.048 \\
Hydraulic diameter $\left(\mathrm{D}_{\mathrm{h}}\right)$ & 0.016 & 0.0192 \\
Length at inlet $\left(\mathrm{L}_{\mathrm{in}}\right)$ & 0.41 & 0.41 \\
Length at outlet $\left(\mathrm{L}_{\text {out }}\right)$ & 0.2 & 0.2 \\
\hline \hline
\end{tabular}

from the sidewall to the mid-plane and to the other wall (to check the symmetry of the results), and from the bottom wall to the top wall. A Phantom v311 camera was placed perpendicular to the illuminated area to record the smoke stream traces in the illuminated slice. The exposure time and the frame rate were adjusted to enable time-averaged flow visualization with the injected smoke.

The light sheet thickness, approximately $1 \mathrm{~mm}$, produced an uncertainty in the sheet location of approximately $0.5 \mathrm{~mm}$. To minimize this error, sharp optics with very a small field depth were selected, therefore leaving only the initial slice position uncertain.

Inevitably, the images obtained included several types of defects generated by non-uniform light intensity and the imperfection of the optical elements and their position. Therefore, special image processing was required to enhance image quality before carrying out any analysis. Local image enhancement was performed via local contrast improvement by local remapping of the grey levels in the vicinity of each pixel.

The channel dimensions were as follows: step size, $s=0.006 \mathrm{~m}$; inlet channel height, $h=$ $0.006 \mathrm{~m}$; exit channel height, $H=h+s=0.012 \mathrm{~m}$; and channel width, $W=0.024 \mathrm{~m}$. The expansion ratio is defined as $E R=H / h$, and the aspect ratio is defined as $A R=W / h$. With these values, the desired expansion ratio of 2 and the desired aspect ratio of 4 were achieved. The length of the inlet channel, $\mathrm{L}_{\mathrm{in}}=0.41 \mathrm{~m}$, was sufficient for flow to develop in the step at $\mathrm{Re}_{\mathrm{Dh}}=850$, according to the equation proposed by Kays et al. ${ }^{26}$

$$
\frac{L_{i n}}{h}=0.05 \cdot R e_{D} \cdot D_{h},
$$

where $\mathrm{D}_{\mathrm{h}}$ is the hydraulic diameter and $\mathrm{Re}_{\mathrm{D}}$ the Reynolds number based on the hydraulic diameter and the duct inlet velocity. The channel length behind the step, $\mathrm{L}_{\mathrm{out}}=0.2 \mathrm{~m}$, ensures that the outlet condition does not affect the flow near the step: Demuren and Wilson ${ }^{27}$ demonstrated numerically that the flow field is not affected by increasing $\mathrm{L}_{\text {out }} / \mathrm{H}$ beyond a value of 7 (a value of 16.66 was used in the current study). The effect of the outflow boundary conditions on flow was studied by Torregrosa et al. $^{28}$

Another channel with an AR of 8 was also used. This second arrangement featured a channel width of $\mathrm{W}=0.048 \mathrm{~m}$, with the rest of the dimensions being the same as those of the AR $=4$ channel. The geometrical parameters of the two experimental arrangements are summarized in the Table I.

\section{B. Effect of Re and aspect ratio}

Flow visualization experiments were performed in the channel with $\mathrm{AR}=4$ for Reynolds numbers ranging from 50 to 1000 . Hereinafter, the same definition for the Reynolds number based on the step height employed in the work of Armaly et al. ${ }^{13}$ is used,

$$
R e_{h}=\frac{\rho_{0} u_{0} 2 h}{\mu},
$$

where $u_{0}$ is the channel inlet velocity, assumed to be uniform, and $h$ is the inflow channel height, which is equal to the step size, $s$, because $\mathrm{ER}=2$. The average inlet flow velocity, $\mathrm{u}_{0}$, is determined 
from the continuity equation, $u_{\mathrm{o}}=\dot{m} / \rho_{\mathrm{o}} A$, where A is the cross-sectional area of the upstream channel. It is worth noting that the relation between the Reynolds number based on the step size, $\mathrm{Re}_{\mathrm{h}}$, and the Reynolds number based on the channel hydraulic diameter, $\operatorname{Re}_{\mathrm{Dh}}$, is

$$
\operatorname{Re}_{h}=\frac{A R+1}{A R} \operatorname{Re}_{D h}
$$

For the sake of simplicity in the notation, the subindex $h$ will be dropped in the rest of the manuscript, this means that all the references to the Reynolds number, Re (without adding any subindex), are referring to $\mathrm{Re}_{\mathrm{h}}$. Flow visualization was conducted in the aforementioned xy and xz planes, aligned with the streamwise direction. The separation between consecutive slices was $1 \mathrm{~mm}$, taking the wall as the starting point. Only the most representative results are shown in the following figures.

Figures 3 and 4 show averaged streamlines for Reynolds numbers ranging from $\mathrm{Re}=100$ to 900 in xy planes, at a $\mathrm{z}$-coordinate of $0.001 \mathrm{~m}$ away from the sidewall, and at the mid-plane, respectively. To facilitate the interpretation of the results, the dividing streamlines have been marked in the figures.

Figure 3 is a representative of the formation of a number of flow features induced by the vicinity of the sidewall. In particular, the presence of the sidewall next to a sudden expansion induces flow going from the wall to the mid-plane, which is commonly known as a wall-jet. In the top wall downstream of the step, a secondary separation zone appears, which does not penetrate up to the mid-plane, and it can be readily observed at a Reynolds number of 200. Although there is no flow recirculation in that region, at a Reynolds number of 100 , a flow distortion near the upper wall can be observed. However, as the Re increases, the formation of a secondary recirculation becomes evident. This sidewall recirculation region becomes larger and is displaced downstream as the Reynolds number increases. Several authors have reported the existence of this bubble (Chiang and Sheu, ${ }^{16}$ Tylli et al. ${ }^{17}$ and Malamataris ${ }^{18}$ ).

Figure 4 presents the flow behavior at the channel mid-plane. The primary or main recirculation zone, behind the step, is larger at the mid-plane than at the region close to the lateral wall, and its length increases with the Reynolds number. The core of the main recirculation region has been marked in the figure. This region is formed because of the blockage effect created by the upper recirculation bubble appearing close to the sidewalls, which, as previously mentioned, does not appear at the mid-plane. This effect increases with Reynolds number.

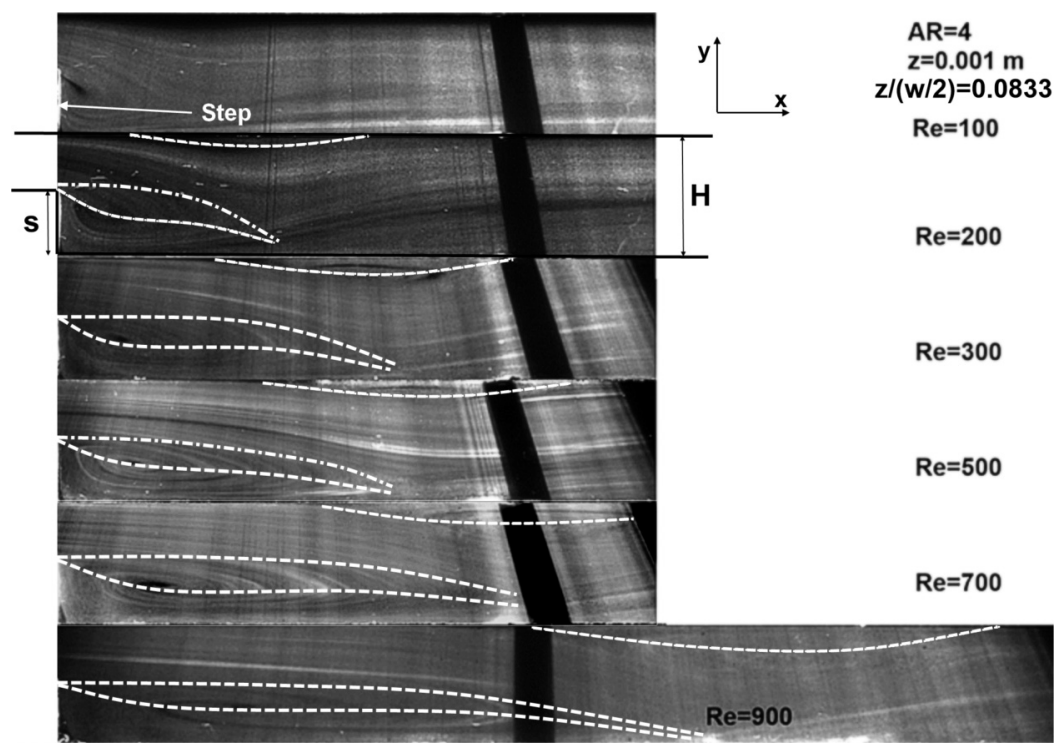

FIG. 3. Flow visualization of $\mathrm{AR}=4$ channel at different $\mathrm{Re}$ at $1 \mathrm{~mm}$ from the lateral wall $(\mathrm{z}=0.001 \mathrm{~m})$. Note the evolution of the bubble downstream, the step, and the secondary bubble at the top wall. Separating streamlines and the primary bubble contour are marked in the figure. 


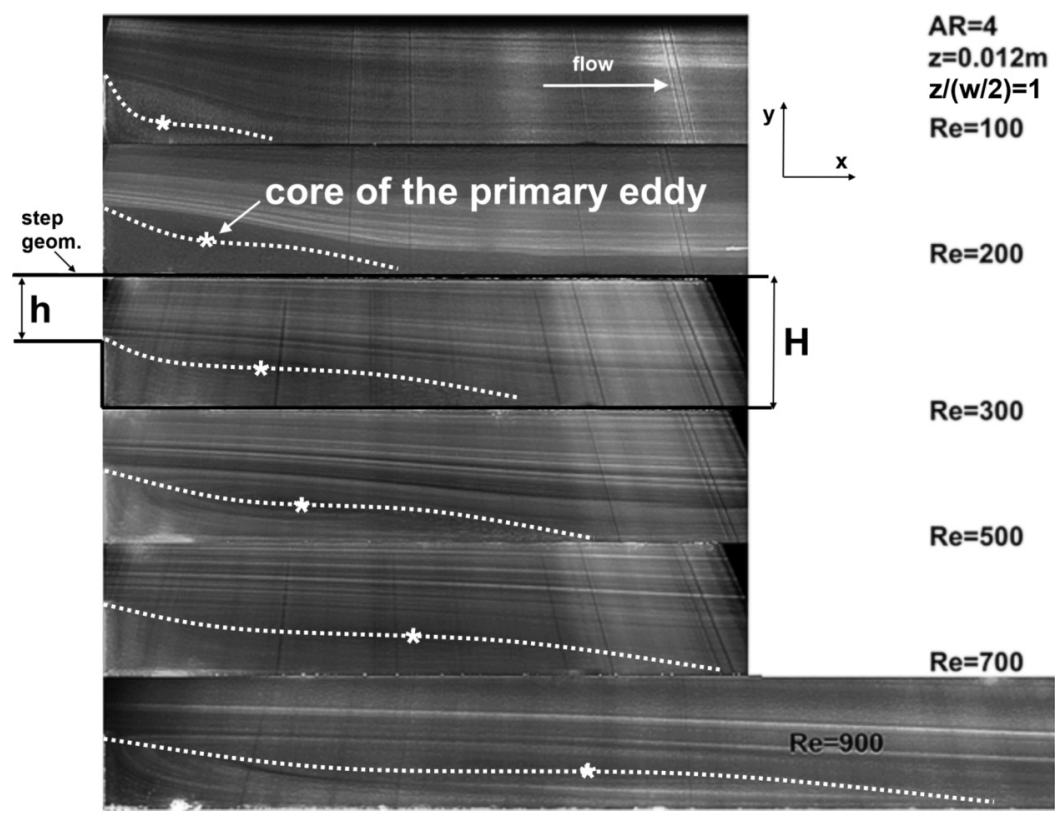

FIG. 4. Flow visualization of $\mathrm{AR}=4$ channel at different $\mathrm{Re}$ at channel mid-plane $(\mathrm{z}=0.012 \mathrm{~m})$. The core of the primary eddy has been marked in the figures. Note the evolution of the bubble downstream, the step, and the absence of a secondary bubble at the top wall.

Horizontal planes at different heights (y-coordinate) behind the step are presented in Figure 5 for a Reynolds number of 500. Although the upper recirculation zone adjacent to the sidewall is limited to a region smaller than the step height, $s$, the three-dimensional effects caused by the sidewall extend through nearly the entire channel span at regions close to the bottom wall. These three-dimensional flows, called wall-jets, also appeared weakly in the experiments performed at Reynolds numbers as low as 100 , at which the upper recirculation bubble did not appear. In planes close to the top wall, these wall-jets disappeared and the secondary recirculation zone emerged at a depth lower than the step height.

For the channel with $\mathrm{AR}=8$, tests were performed at two different Reynolds numbers, 300 and 500. The results are analogous to those obtained at $\mathrm{AR}=4$, but with relevant changes in size (length, width, and depth) of the secondary recirculation bubble. This recirculation was generally larger at $\mathrm{AR}=8$ than at $\mathrm{AR}=4$, as observed by comparing the flow in Figure 3 with that in Figure 6.

\section{NUMERICAL MODELS}

In the present work, compressible three-dimensional airflow over a backward-facing step was studied numerically. The flow was modeled by the compressible Navier-Stokes equations for transient viscous fluid flow. The governing equations are

$$
\begin{gathered}
\frac{\partial \rho}{\partial t}+\frac{\partial}{\partial x_{i}}\left(\rho u_{i}\right)=0 \\
\frac{\partial\left(\rho u_{i}\right)}{\partial t}+\frac{\partial}{\partial x_{j}}\left(\rho u_{j} u_{i}\right)=-\frac{\partial p}{\partial x_{i}}+\frac{\partial \tau_{i j}}{\partial x_{j}}+\rho g
\end{gathered}
$$

where $u$ is the fluid velocity, $\rho$ the fluid density, $p$ is the static pressure, and $\tau_{i j}$ the viscous stress tensor.

The range of flows studied are in the laminar regime, $\mathrm{Re}=[100-1000]$, which lies very close to the transitional regime. The governing equations were solved in combination with an equation of state for the flow, such as the ideal gas law. The distinction between laminar, transitional, and 


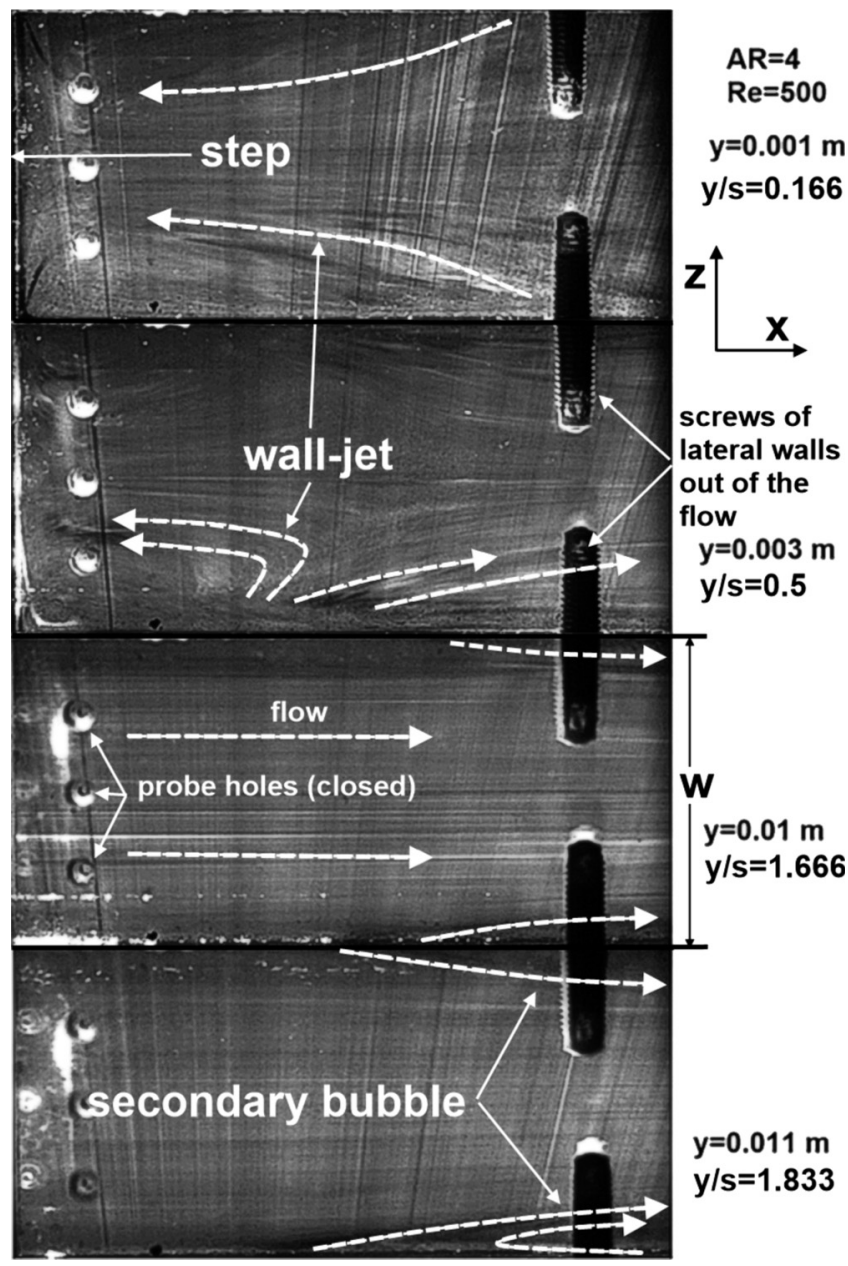

FIG. 5. Flow visualization of $\mathrm{AR}=4$ channel in horizontal planes at different heights $(0.001,0.003,0.01$, and $0.011 \mathrm{~m})$. Flow direction, wall-jets, and separating streamlines have been marked in the figure.

turbulent flow is not straightforward. Sometimes a flow appears in different states depending on the area of the analysis domain that it is observed. The general problem concerning the transition from laminar to turbulent flow, that is, the computation of the onset of transition as well as the length of the transition zone, is a common subject of fundamental research today.

The classical unsteady Reynolds-averaged Navier-Stokes (URANS) equations do not have, in certain situations, enough accuracy for such flows even when advanced high-order turbulence models are used for closure because of the underlying assumption that flows are fully turbulent. It is important to note again that the analysis performed in the present work is close to the laminar

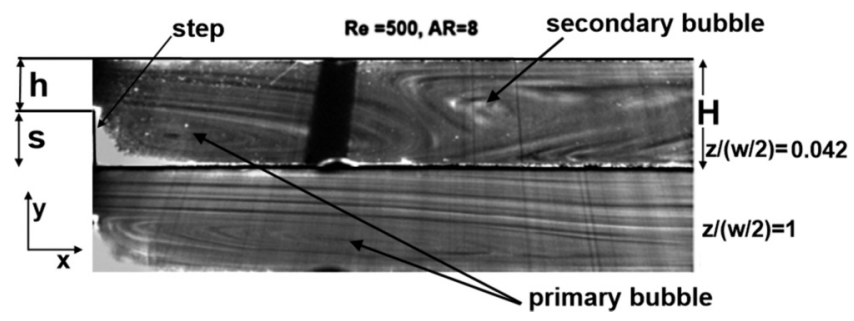

FIG. 6. Flow visualization of $\mathrm{AR}=8$ channel at $\mathrm{Re}=500$. Two vertical planes are shown: a plane at $1 \mathrm{~mm}$ from the sidewall and the mid-span of the channel. The main flow characteristics may be compared with those shown in Figure 3 for the $A R=$ 4 channel. 
limit of the flow. Solving the problem using a direct numerical solution (DNS) is the most reliable procedure because all turbulent scales are resolved both spatially and temporally, but this accurate numerical method requires substantial computational resources.

The third approach, large eddy simulation (LES), lies between URANS and DNS. The equations are filtered to separate large eddies from small ones, and the large geometrically dependent eddies are resolved on grid scales, whereas the effects of the smaller scales are modeled using a sub-grid scale (SGS) model. LES requires lower resolution than that required by DNS and is more accurate than RANS. Hence, LES is a promising tool for understanding the physics of flows in cases in which both the laminar and transitional regimes are present (Ferziger and Peric ${ }^{29}$ and Lesieur et al. ${ }^{30}$ ).

The LES approach was first used by Smagorinsky ${ }^{31}$ and Lilly. ${ }^{32}$ Nevertheless, the SGS model based on the eddy-viscosity model proposed by Smagorinsky ${ }^{31}$ has difficulties in reproducing transitional laminar flow and near wall flow. The original Smagorinsky model is too dissipative, distorting laminar flow and relaminarizing transitional flow, which occurs because the fixed value of the Smagorinsky constant, $\mathrm{C}_{\mathrm{s}}$, in the model, must be tuned to precisely characterize transitional flow. Commonly used values can dampen large-scale fluctuations in the presence of mean shear and in transitional flows near a solid boundary (Zang et al. ${ }^{33}$ ).

A more suitable SGS model for simulating transition should be able to address laminar, transitional, and turbulent flows equally well, while leaving the laminar flow unaffected. The dynamic procedure proposed by Germano et al. ${ }^{34}$ represent a major advance in the development of LES models. In the authors' dynamic Smagorinsky model, the $\mathrm{C}_{\mathrm{s}}$ constant is not specified $a$ priori but rather computed based on local flow conditions. Thus, it is possible to simulate the laminar-turbulent transition using Germano's dynamic model. ${ }^{34}$

In the present work, the dynamic Smagorinsky model was used to solve the Navier-Stokes equations

$$
\begin{gathered}
\frac{\partial \rho}{\partial t}+\frac{\partial}{\partial x_{i}}\left(\rho u_{i}\right)=0 \\
\frac{\partial\left(\rho \overline{u_{i}}\right)}{\partial t}+\frac{\partial}{\partial x_{j}}\left(\rho \overline{u_{i} u_{j}}\right)=-\frac{\partial \bar{p}}{\partial x_{i}}+\frac{\partial}{\partial x_{j}} \mu_{e f f}\left(\frac{\partial \overline{u_{i}}}{\partial x_{j}}+\frac{\partial \overline{u_{j}}}{\partial x_{i}}\right),
\end{gathered}
$$

where $\mu_{\text {eff }}$ is the effective viscosity, i.e., the molecular viscosity plus the eddy viscosity, defined by the Smagorinsky model as follows:

$$
\mu_{e f f}=\mu+\rho\left(C_{s} \Delta\right)^{2} \sqrt{2 S_{i j} S_{i j}},
$$

where $\mathrm{S}_{\mathrm{ij}}$ represents the strain tensor and $\Delta=(\delta \mathrm{x} \delta \mathrm{y} \delta \mathrm{z})^{1 / 3}$ is the filter width. Details of the procedure used to compute $\mathrm{C}_{\mathrm{s}}$ can be found in the study by McGrattan et al. ${ }^{35}$

The abovementioned governing equations were solved using two well-established computational fluid dynamics codes, the commercial CFD package (ANSYS-Fluent ${ }^{36}$ ) and an open source code (FDS-NIST ${ }^{35}$ ) for validation. The two codes use different approaches for the equation of state: In ANSYS-Fluent, the ideal gas law is imposed, whereas in FDS a low Mach number approximation is applied.

Although the use of LES for laminar flow simulations is not an uncommon practice in the literature, a numerical experiment was performed for Reynolds number 100 and the channel with an $\mathrm{AR}=4$ comparing the obtained results with the code FDS-LES using the dynamic Smagorinsky model (grid size $3.84 \times 10^{6}$ ) and FDS-DNS (grid size $5 \times 10^{7}$ ). No difference could be detected between the results of the two methods, supporting the use of LES for laminar regime.

Additionally, the results were compared and validated with published experimental results. As previously mentioned, the flow was modeled with the compressible flow equations; however, for the operating conditions of these tests (low flow velocity), the flow is quasi-incompressible and therefore the numerical model can be validated using published experimental results for incompressible flow.

\section{A. Description of CFD codes}

FDS numerically solves a form of the Navier-Stokes equations for low Mach numbers. The core algorithm is an explicit predictor-corrector scheme, second order accurate in space and time. 
Although LES was used in the present work, it is possible to perform DNS if the underlying mesh is sufficiently fine as it was previously mentioned.

The time step selection is based on the flow speed within a particular mesh. The well-known Courant-Friedrichs-Lewy (CFL) constraint, used in explicit schemes as a stability criterion to maintain physically realizable conditions, states that

$$
C F L=\delta t \frac{\|u\|}{\delta x}
$$

where

$$
\frac{\|u\|}{\delta x}=\max \left(\frac{\left|u_{x}\right|}{\delta x}, \frac{\left|u_{y}\right|}{\delta y}, \frac{\left|u_{z}\right|}{\delta z}\right) .
$$

FDS selects the time step size to ensure that the CFL is smaller than one. Physically, the criterion states that a fluid element should not traverse more than one cell within a time step. The governing equations are solved on a uniformly spaced three-dimensional grid of rectangular cells.

ANSYS-Fluent solves the full Navier-Stokes equations in two-dimensional or three-dimensional geometries, discretizing equations on a general grid, even in a non-uniform one, via the finite volume approach. ${ }^{35}$ An implicit solver was used in conjunction with an algebraic multigrid method. For the transient formulation, two different approaches were followed: iterative and non-iterative time advancement. For the former approach, the SimpleC scheme was adopted as a pressure-velocity coupling scheme, and the momentum equations were discretized with the bounded central differencing scheme. The latter was performed using a pressure implicit with splitting operator (PISO) scheme for pressure-velocity coupling (Issa et $_{\text {al }}{ }^{37}$ ). In both cases, a bounded second order implicit temporal discretization was used with a typical time increment of $10^{-5} \mathrm{~s}$. As an illustration, the maximum CFL in the $\mathrm{Re}=700$ simulation with the final mesh used in this work was approximately 0.3 . The convergence criteria were set such that the scaled residuals must be smaller than $10^{-5}$ for the mass and velocity, and the convergence was ensured by using the default under-relaxation factors.

The dynamic Smagorinsky procedure in both Fluent and FDS is based on work of Germano et al. ${ }^{34}$ In ANSYS-Fluent, in particular, the procedure is executed using the development of $\mathrm{Kim}^{38}$ for non-structured grids.

The problem is solved in a three-dimensional domain that corresponds to the extent of the geometry of the experimental apparatus depicted in Figure 2. The origin of the coordinate system is located at the lower corner of the stepped wall with the $\mathrm{x}$-axis oriented along the flow direction and the $y$-axis aligned with the vertical direction. The computational space was discretized using a hexahedral mesh designed with a near-wall distance $y^{+} \approx 1$. As a characteristic figure, the value of $y+$ for the final mesh used in ANSYS-Fluent in the $\mathrm{Re}=1000$ case was 0.35 for the lower wall and 1.4 for the lateral and upper walls. In FDS, the values were slightly higher because the mesh was uniform and thus had no refinement near the walls; in this case the y+ value was found to be smaller than 2 , and in the step region, the value was approximately 1.4. A mesh size sensitivity study was conducted as explained in Section III B.

The boundary conditions used for this computational domain consisted of an air stream with uniform temperature, $\mathrm{T}_{0}$, and velocity, $\mathrm{u}_{0}$, profiles at the inlet. A pressure outlet condition was applied as the boundary condition at the exit plane (zero gauge pressure). As previously mentioned, the exit plane was located sufficiently downstream from the step to minimize the influence of the outflow conditions on the results in the region of interest. In fact, several numerical tests were conducted in the case of a low aspect ratio, $\mathrm{AR}=4$, for two different Reynolds numbers, 700 and 1000. These computations confirmed that over the range of Reynolds numbers investigated, a longer domain did not affect the flow in the step region, as shown in Figure 7 (Figure 7(a) for $\mathrm{Re}=1000$ and Figure 7(b) for $\mathrm{Re}=700$ ) and had a limited effect at Reynolds numbers slightly greater than 1000 .

A non-slip wall condition was considered at the horizontal walls of the domain and the step. To isolate the three-dimensional effects induced by the sidewall and those due to flow turbulence, two different conditions were considered along the lateral walls: first, a non-slip boundary condition was used, and afterwards, a slip wall boundary condition was considered. 

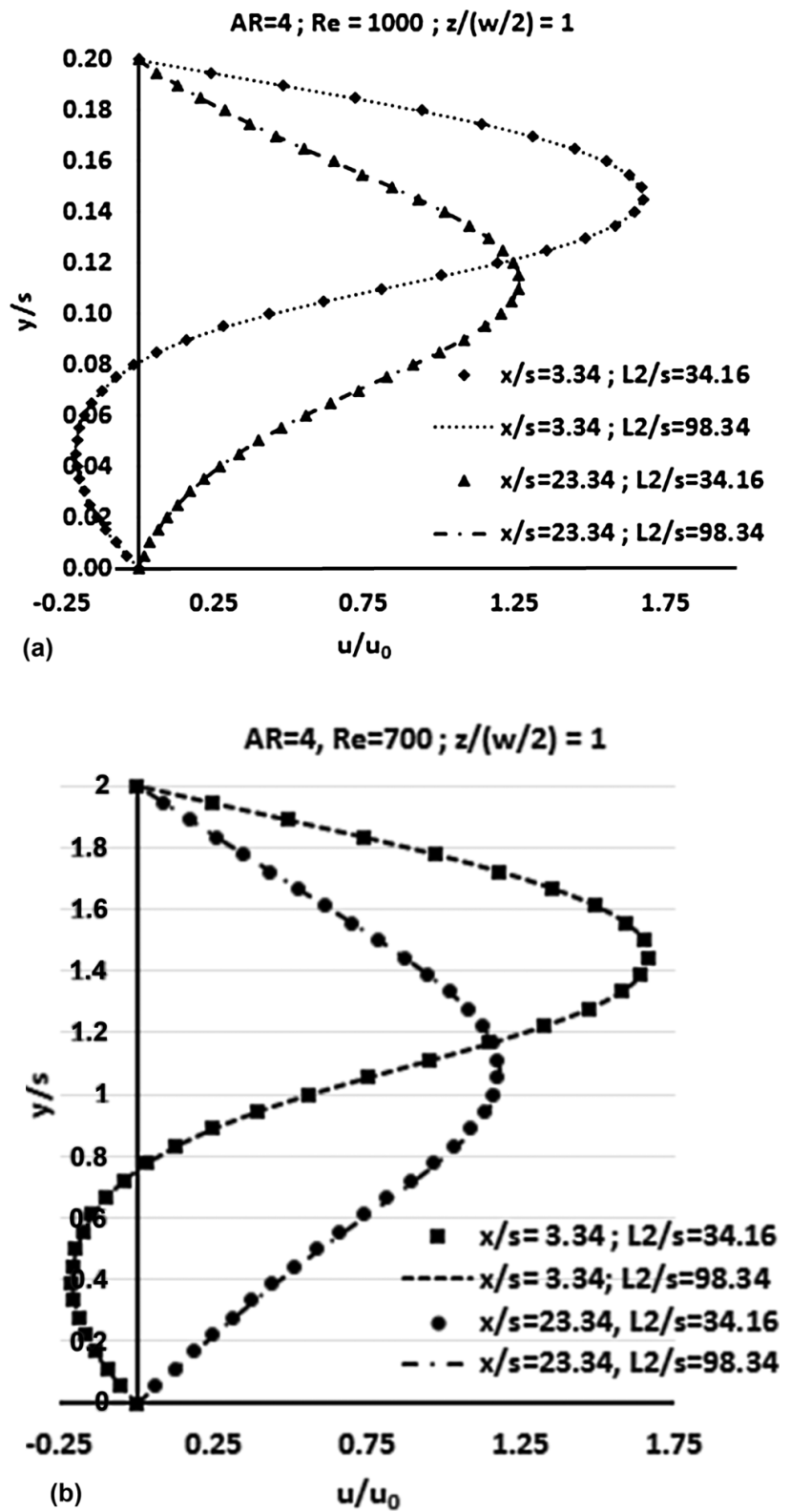

FIG. 7. The effect of the length of the domain downstream of the step was analyzed. No variation in the velocity profiles was observed between two different longitudinal positions $(x / s=3.34$ and $x / s=23.34)$ and between two Reynolds numbers, 1000 (a) and 700 (b).

\section{B. Mesh-independence analysis}

For both codes, ANSYS-Fluent and FDS, a mesh-independence analysis was performed to ensure the independence of the results with respect to the number of cells; therefore, five hexahedral meshes were considered with a similar number of cells between the two codes. The independence analysis was performed for the case of $\mathrm{AR}=4$ channel. The meshes compared in the study are summarized in Table II. To provide an approximation idea of the cell size in each direction (streamwise, vertical, and spanwise), for the M3 and M5 FDS meshes, the number of cells in each direction was 1600,40 , and 60 cells and 2800, 54, and 108 cells, respectively. In the case of ANSYS-Fluent, the M3 mesh was generated using a fixed inflation with the following characteristics: initial element height $0.05 \mathrm{~mm}$, cell growth size ratio 1.2 , and maximum element size $0.65 \mathrm{~mm}$, whereas the M5 was generated with an initial size of $0.02 \mathrm{~mm}$ and a maximum size of $0.35 \mathrm{~mm}$. 
TABLE II. Different meshes considered in the study.

\begin{tabular}{lrr}
\hline \hline Mesh & FDS & \\
\hline M1 & 1800000 & ANSYS-Fluent \\
M2 & 2721600 & 1808496 \\
M3 & 3840000 & 2449005 \\
M4 & 6750000 & 3827250 \\
M5 & 16329600 & 6823454 \\
\hline \hline
\end{tabular}

Velocity profiles in the mid-plane and a plane close to the sidewall at different $\mathrm{x}$-positions and for $\mathrm{Re}=700$ and meshes M1-M4 were analyzed. Additionally, because of the influence of Re on the transitional flow, an additional study was performed with a finer mesh (defined in Table II as M5) and for a higher Reynolds number, $\mathrm{Re}=1000$. A summary of the results obtained is presented in Table III and Figure 8. The figure shows the main characteristic lengths of the problem (as depicted in Figure 2) at the mid-plane of the channel.

Figure 8 presents the velocity profiles at several locations and distances from the step using the two codes considered in this work. Figures 8 (a) and 8(b) present the velocity profiles for $\operatorname{Re}=700$
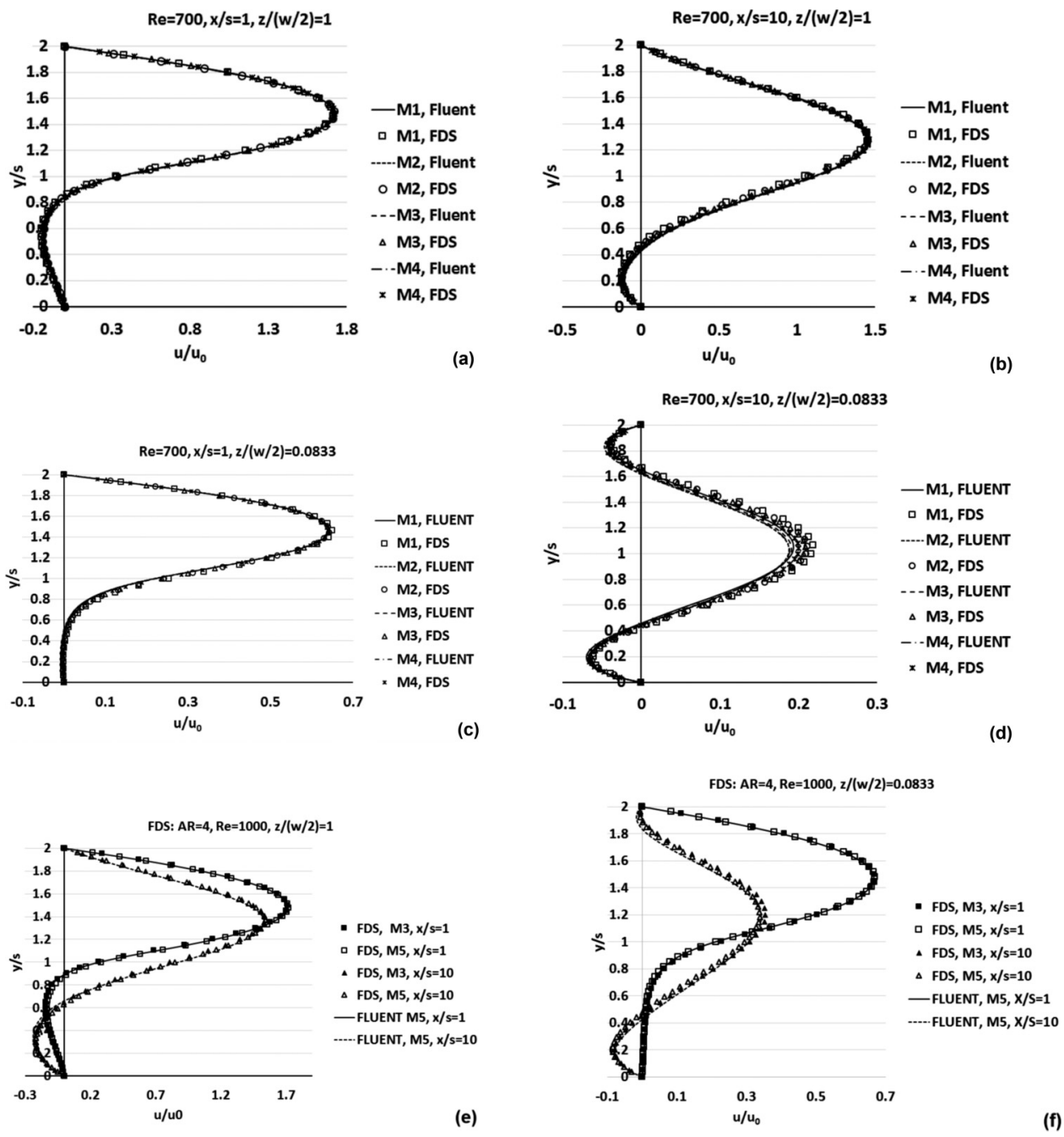

FIG. 8. Velocity profiles in the mid-plane at several distances from the step obtained with FDS and ANSYS-Fluent in different locations for the different meshes. For $\operatorname{Re}=700$ : (a) and (b) at the mid-plane; (c) and (d) in a plane close to the side-wall; and for $\operatorname{Re}=1000$ : (e) and (f). 
and for meshes M1-M4 in the mid-plane at two different streamwise locations $\mathrm{x} / \mathrm{s}=1$ and $\mathrm{x} / \mathrm{s}=10$, respectively. All of the profiles in the mid-plane appear to overlap onto a single curve. Figures 8(c) and $8(\mathrm{~d})$ show the velocity profiles for the same cases but contained within a plane at a distance of $1 \mathrm{~mm}$ from the lateral wall. In this case, the results of FDS show slight differences, likely because the mesh used in computations with FDS was uniform. Finally, the mesh-independence analysis has been extended to a finer mesh (M5) and a higher Reynolds number $(\operatorname{Re}=1000)$ because these values represent a more critical situation. Figures $8(\mathrm{e})$ and $8(\mathrm{f})$ present the velocity profiles obtained from this analysis. The figures compare the profiles obtained with the M5 mesh using the two codes with those obtained with the M3 mesh in FDS in the different previously mentioned locations. The results show that no significant difference was introduced by increasing the mesh size by a factor of nearly 4; thus, the increase in computational cost is not justified.

To quantitatively evaluate the difference between the profiles, a procedure similar to that described by Wang et al. ${ }^{39}$ was performed. The procedure was based on evaluating the root-meansquare error in the velocity, that is,

$$
\varepsilon=\sqrt{\frac{1}{N} \sum_{i=1}^{N}\left(\frac{V_{i, n-1}-V_{i, n}}{V_{a v e, n}}\right)},
$$

where $V_{i, n-1}$ is the velocity in the former grid, $V_{i, n}$ is the velocity in the current grid, subscript i denotes the i-th sampling point, $V_{a v e}$ is the mean velocity of the profile, and $N$ is the number of sampled points. This procedure was applied to the velocity profiles at the mid-plane at a distance $\mathrm{x} / \mathrm{s}$ $=1$ from the step $\left(\varepsilon_{1}\right)$ and to the velocity profile in a plane close to the wall at a distance $\mathrm{x} / \mathrm{s}=10$ $\left(\varepsilon_{2}\right)$. The results are presented in Table IV.

Based on these results, mesh M3 was considered for the rest of the computations performed in this work, in which the root-mean-square error in the velocity was less than $0.5 \%$. Finally, a mesh with $3.8 \times 10^{6}$ grid cells was used in the computations in the AR $=4$ case. The number of cells in the $\mathrm{AR}=8$ domain was increased proportional to its volume, i.e., approximately twice as many cells.

TABLE III. Values of the main characteristic lengths of the problem: $x_{u}$ is the reattachment length of the primary bubble, $x_{2}$ the position of the detachment, $x_{3}$ the location of the reattachment of the secondary bubble length, and y depth (in the vertical direction) of the bubble.

\begin{tabular}{|c|c|c|c|c|}
\hline \multicolumn{5}{|c|}{ Fluent $(\operatorname{Re}=700)$} \\
\hline & $\mathrm{x}_{\mathrm{u}} / \mathrm{s}$ & $\mathrm{x}_{2} / \mathrm{s}$ & $\mathrm{x}_{3} / \mathrm{s}$ & $\mathrm{y} / \mathrm{s}$ \\
\hline M1 & 14.013 & 6.1599 & 19.071 & 0.4021 \\
\hline M2 & 13.8683 & 6.0885 & 18.8661 & 0.4194 \\
\hline M3 & 13.9138 & 6.1391 & 19.078 & 0.4246 \\
\hline M4 & 13.9045 & 6.1123 & 19.1918 & 0.4272 \\
\hline \multicolumn{5}{|c|}{$\operatorname{FDS}(\operatorname{Re}=700)$} \\
\hline M1 & 14.164 & 6.9254 & 18.6198 & 0.38741 \\
\hline M2 & 14.3095 & 6.8073 & 18.82 & 0.39638 \\
\hline M3 & 14.1505 & 6.7624 & 18.9716 & 0.40325 \\
\hline M4 & 14.118 & 6.6361 & 19.1502 & 0.41661 \\
\hline \multicolumn{5}{|c|}{ Fluent $(\operatorname{Re}=1000)$} \\
\hline & $\mathrm{x}_{\mathrm{u}} / \mathrm{s}$ & $\mathrm{x}_{2} / \mathrm{s}$ & $\mathrm{x}_{3} / \mathrm{s}$ & $\mathrm{y} / \mathrm{s}$ \\
\hline M3 & 19.90 & 9.5167 & 27.3216 & 1.5645 \\
\hline M5 & 19.85 & 9.3567 & 27.1676 & 1.5659 \\
\hline \multicolumn{5}{|c|}{$\operatorname{FDS}(\operatorname{Re}=1000)$} \\
\hline M3 & 20.233 & 9.5998 & 27.4666 & 1.5765 \\
\hline M5 & 19.883 & 9.4833 & 27.3166 & 1.5534 \\
\hline
\end{tabular}


TABLE IV. Results of the mesh independence analysis for both codes for Re 700.

\begin{tabular}{|c|c|c|c|c|c|c|c|}
\hline \multicolumn{4}{|c|}{ FDS } & \multicolumn{4}{|c|}{ ANSYS-Fluent } \\
\hline Mesh & Cells & $\varepsilon_{1}$ & $\varepsilon_{2}$ & Mesh & Cells & $\varepsilon_{1}$ & $\varepsilon_{2}$ \\
\hline M1 & 1800000 & 0.01028 & 0.00686 & M1 & 1808496 & 0.00945 & 0.01594 \\
\hline M2 & 2721600 & 0.00587 & 0.00335 & M2 & 2449005 & 0.00627 & 0.00651 \\
\hline M3 & 3840000 & 0.00413 & 0.00320 & M3 & 3827250 & 0.00422 & 0.00423 \\
\hline M4 & 6750000 & $\ldots$ & $\ldots$ & M4 & 6823454 & $\ldots$ & $\ldots$ \\
\hline
\end{tabular}

\section{Model validation (comparison with results from Armaly et al.)}

The validity of the numerical models used with the two codes was verified by direct comparison with the experimental results ${ }^{13}$ in the channel with $A R=8(D=8 h)$ and for a Reynolds number of $\operatorname{Re}=343$, computed according to the definition used by Armaly et al., ${ }^{13}$ i.e., based on the channel height $(2 h)$. Streamwise velocity profiles in the mid-plane at several distances from the step obtained with the two codes and compared with experimental data are presented in Figure 9. Good agreement was achieved at $\mathrm{x} / \mathrm{s}=0$ (Figure $9(\mathrm{a})$ ) and $\mathrm{x} / \mathrm{s}=1$, and very slight discrepancies were observed at $\mathrm{x} / \mathrm{s}=7$ (Figure 9(b)). At this point, regarding the comparison of the numerical and experimental data, it is necessary to note that the experimental data reported by Armaly et al. ${ }^{13}$ were digitized based on the figures presented in the paper because tabular results were unavailable. In addition, there was uncertainty regarding the location of the laser Doppler velocimetry (LDV) system and velocity measurement of the experimental results. The reattachment line was computed

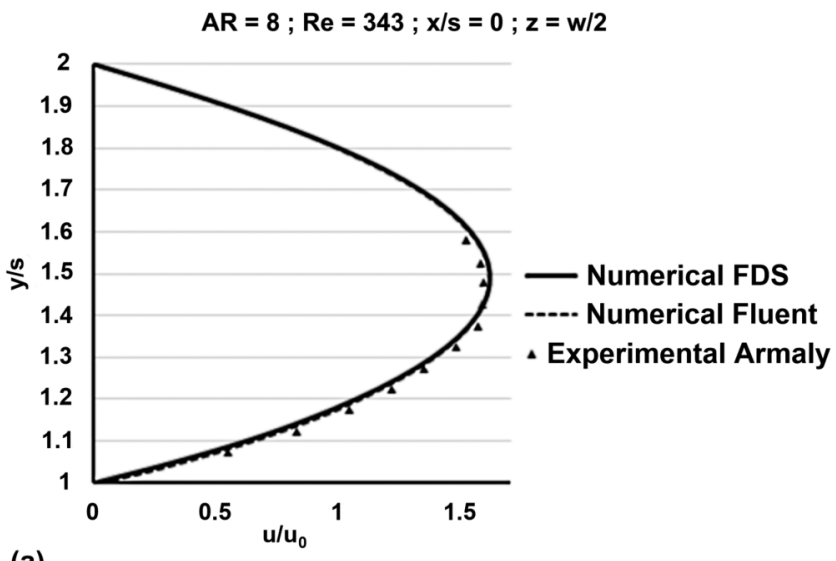

(a)

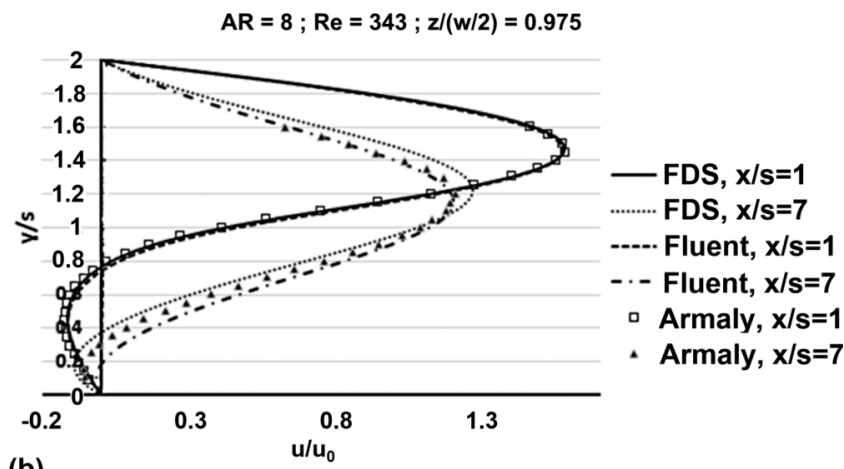

(b)

FIG. 9. Streamwise velocity profiles in the mid-plane at several locations from the step obtained with the two codes and comparison with experimental data. (a) is the profile at the step $(\mathrm{x} / \mathrm{s}=0)$ and $(\mathrm{b})$ includes two streamwise positions $(\mathrm{x} / \mathrm{s}=1$ and $\mathrm{x} / \mathrm{s}=7)$. 


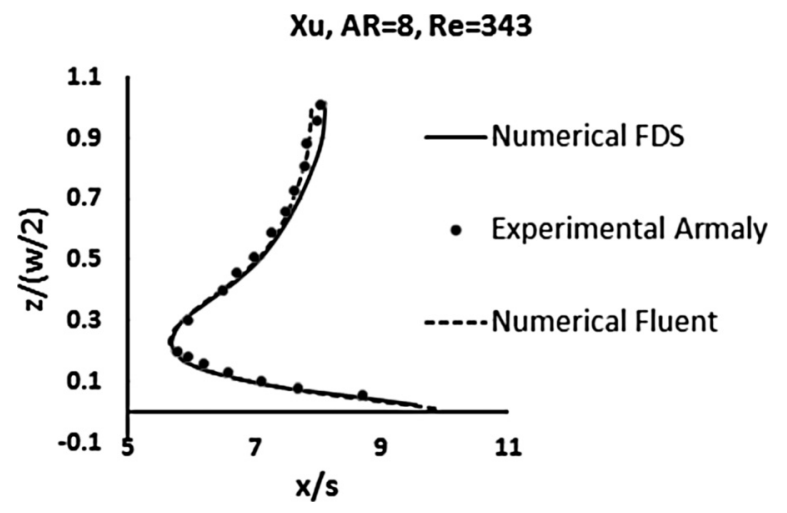

FIG. 10. Reattachment length computed in agreement with the definition reported by Armaly et al. ${ }^{13}$ The numerical results obtained with the two codes are in good agreement with the experimental measurements.

according to the work of Armaly et al. ${ }^{13}$ as the region where the mean streamwise velocity component changed sign from positive to negative at a plane adjacent and parallel to the bottom wall. The results were compared with experimental measurements; the comparison is presented in Figure 10 for the same $\mathrm{y} / \mathrm{s}$ value. Again, the agreement was good over the entire span, including the region close to the sidewall. There was only a slight difference in the region close to the sidewall when the uniform mesh of the FDS code was used. The abovementioned agreement lends support to the validity of the codes in the present work.

In addition, the previously mentioned results of the mesh-independence analysis (Section III B) and validation with experimental data (Section III C) allowed for the comparison of the two codes used in this work. The two codes involve different numerical procedures. The velocity profiles obtained, as well as the reattachment length computed with the two codes, were generally very similar, despite using different meshes with roughly the same number of cells and being uniform in FDS, and non-uniform (refined close to the walls) in ANSYS-Fluent. This agreement lends additional support to the validity of the results obtained thereafter.

\section{Comparison of numerical and experimental results}

To gain a deeper understanding of the experimental results obtained in this work, two-dimensional streamtraces in three-dimensional data were obtained, forcing the streamtraces to remain in the slice plane, similarly to the recorded images in the experiment. The simulations discussed in the rest of the present study were performed with both codes, with similar results obtained between them. For the sake of conciseness, only the results obtained with one of the codes are presented.

Figure 11 shows a comparison of numerical and experimental streamlines and iso-contours of time-averaged streamwise velocity for the AR $=4$ channel at Reynolds number of 500 . The post-processing planes are perpendicular to the bottom wall (Figure 11(a)) and planes parallel to the bottom wall (Figure 11(b)) placed at several distances from the wall.

Regarding only the qualitative behavior of the flow, the agreement between the experimental flow visualization and the predicted stream-traces is very good. The upper wall recirculation zone disappears for a spanwise distance over the range of $s / 3-s / 2$ from the lateral wall. The primary recirculation zone in both the experimental and simulated results is longer (in the x-direction) in the mid-plane than in planes closer to the sidewall. The corresponding video recording shows that the flow is symmetrical and steady (because no fluctuations are observed), in agreement with the numerical results obtained for this Reynolds number. This phenomenon was consistently observed in the experiments conducted at Reynolds numbers equal to or less than 1000 and accurately predicted by analysis. Again, it is interesting to note that at a Reynolds number of 100, the upper bubble did not form.

The flow symmetry is shown in Figure 11(b), which shows horizontal (x-z) planes at different heights. Inspection of the slice near the top wall reveals that the sidewall effects distort the flow 

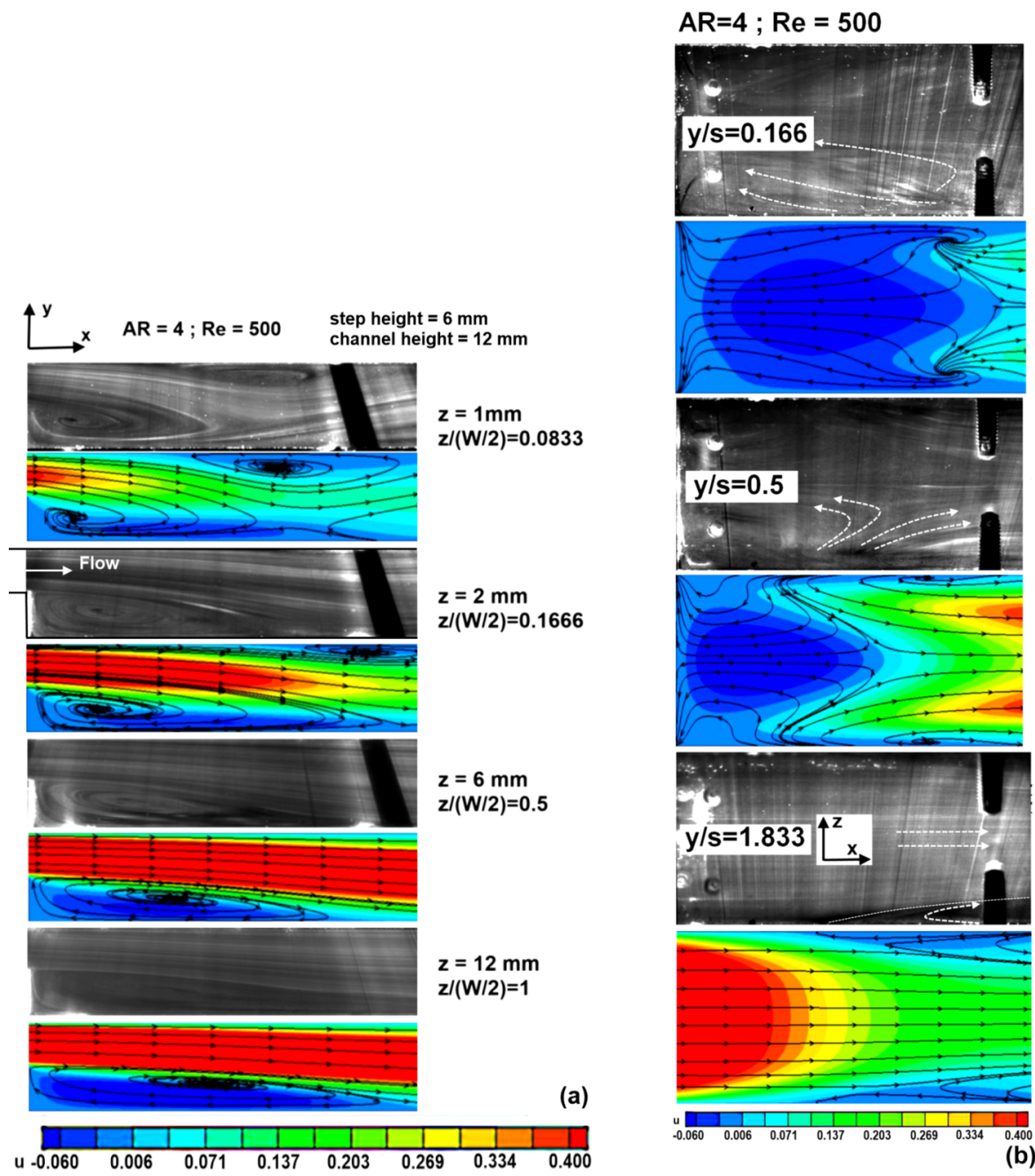

(b)

FIG. 11. Comparison of numerical and experimental stream lines for the $\mathrm{AR}=4$ channel at a Reynolds number of 500 . Numerical results include streamwise velocity contours: x-y planes are presented in (a), and x-z planes are presented in (b).

adjacent to the sidewall in planes close to the upper wall when the secondary recirculation zone appears. Moreover, the sidewall also affects the flow over the entire span because of the existence of the opposite wall-jet. The results obtained confirm that the wall-jet was responsible for the minimum and maximum of the reattachment lengths near the lateral wall.

The effect of the Reynolds number in the secondary recirculation zone next to the sidewall is shown in Figure 12 for the AR $=4$ channel. The bubble began forming at a Reynolds number of 200 and grew in depth in the spanwise direction (perpendicular to the bottom wall) with the Reynolds number, also moving downstream from the step. In any case, for $A R=4$, there was no coalescence of the upper recirculation region with the primary one close to the lateral wall, up to a Reynolds number of 1000. A closer look at the numerical results, which could not be presented in these figures, similarly to the experimental results, reveals that the flow was symmetrical and steady for low and moderate Reynolds numbers, with the exception of small, low-frequency oscillations near the reattachment points for moderate values of the Reynolds number.

An analogous situation could be observed in the AR $=8$ channel. However, the extent of the upper wall recirculation zone was larger than that observed at $\mathrm{AR}=4$. For comparison, the case for $\operatorname{Re}=500$ is presented in Figure 13, where the regions of positive and negative steam-wise velocity are delimited by dashed lines. 

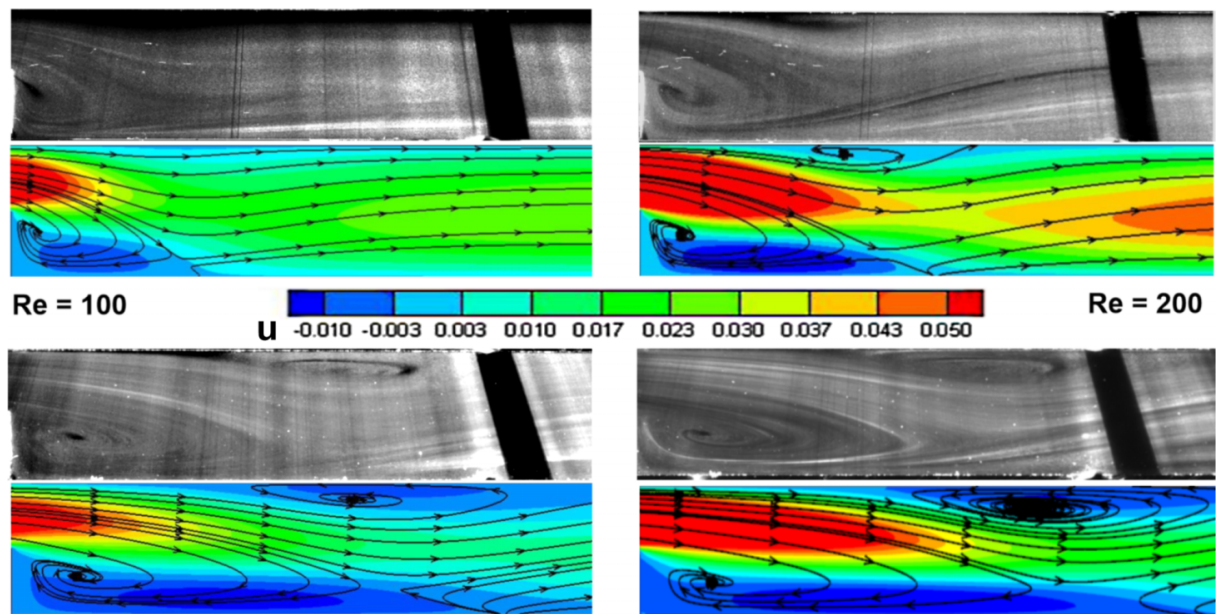

$\operatorname{Re}=\mathbf{2 0 0}$

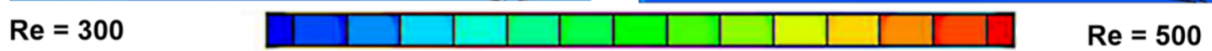

u $-0.020 \cdot 0.0030 .0140 .0310 .048 \quad 0.0650 .0820 .098 \quad 0.1150 .1320 .1490 .1660 .1830 .200$

$\operatorname{Re}=\mathbf{5 0 0}$
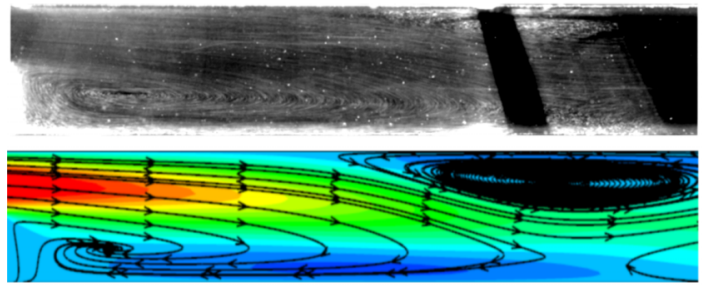

$A R=4$

$z=1 \mathrm{~mm}$

$z /(W / 2)=0.0833$
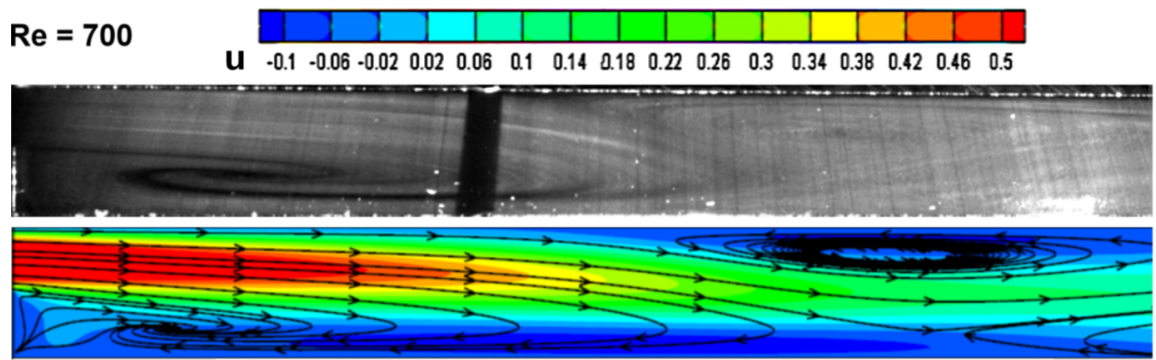

$\operatorname{Re}=1000$

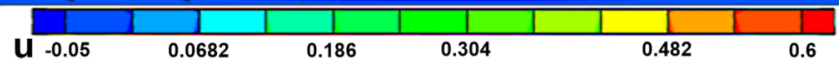

FIG. 12. Effect of Reynolds number in the secondary recirculation zone next to the sidewall for the AR $=4$ channel. Comparison of experimental and numerical results.

\section{ASSESSMENT OF THE SECONDARY BUBBLE AND ITS EFFECT ON THE FLOW}

The main objective of the present work was to assess the sidewall-induced recirculation zone, which is important mainly in channels with a small aspect ratio. A systematic study for $\mathrm{AR}=4$ and $\mathrm{AR}=8$, as a function of Reynolds number, was performed. The study covered three different topics related to the secondary bubble: the effect of the bubble on the reattachment length of the

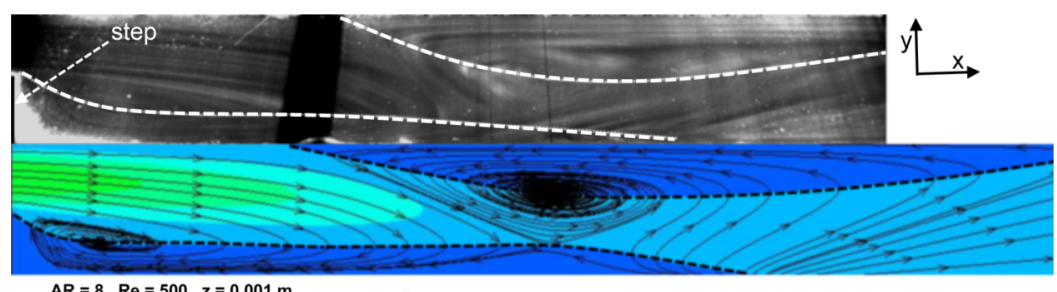

FIG. 13. Secondary recirculation zone in the $\mathrm{AR}=8$ case and at $\mathrm{Re}=500$ at a distance of $1 \mathrm{~mm}$ from the sidewall. 
primary recirculation zone, the effect of the Reynolds number and aspect ratio on the dimensions of the secondary bubble, and the differentiation of the effects directly induced by the wall and the inherent 3D flow features. These topics were addressed by analyzing the flow considering a free-slip boundary condition on the sidewalls instead of a non-slip one.

\section{A. Effect of secondary bubble on primary reattachment length $\left(x_{u}\right)$}

The effect of the secondary eddy on the three-dimensionality of the flow could also be discerned based on the comparison of the reattachment point of the primary eddy at the channel central plane. CFD results for $\mathrm{AR}=4$ and $\mathrm{AR}=8$ channels, in addition to the well-documented experimental and numerical results for large aspect ratios $(A R>30)$ and $2 \mathrm{D}$ and $3 \mathrm{D}$ results obtained from the literature, were compiled. Figure 14 shows the primary eddy dimensionless length at the mid-plane as a function of Reynolds number. The three-dimensional CFD results match the literature data below $\operatorname{Re}=400$. However, for higher Reynolds numbers, the results begin to deviate, and the reattachment length at the mid-plane is always higher than the corresponding two-dimensional or quasi-two-dimensional results obtained for large aspect ratios. It is worth noting that the free-slip case computed in this work presents the same tendencies observed in the results reported by Biswas et al. ${ }^{4}$ and in the 2D cases reported by Iwai et al. ${ }^{19}$ The 3D results reported by Iwai et al. ${ }^{19}$ (with an $\mathrm{AR}=16$ ) are quite similar to the results obtained in the present work, but they only cover a narrow range of Reynolds numbers.

It is notable that the presence of the upper wall recirculation zone adjacent to the sidewall increased the existing wall-jets. These jets enhanced the primary eddy at the mid-plane, therefore increasing the distance from the step wall to the reattachment point in low-aspect-ratio channels. Figure 15(a) presents flow streamlines starting close to the sidewalls upstream of the step for a Reynolds number of 100 and $A R=4$. Areas where the sign of the axial velocity changes are highlighted. The deviation of the flow in the upper zone of the channel at the step and its interaction with the sidewalls yielded swirling flows, thus forming wall-jets. These jets traveled through the channel and served as the source of the maximum and minimum values appearing close to the lateral wall in the spanwise distribution of the primary reattachment length.

The negative pressure gradient that developed downstream of the step due to the sudden expansion was responsible for the secondary eddy that developed close to the sidewall as the Reynolds number increased. Figure 15(b) illustrates this phenomenon, presenting the streamlines for a Reynolds number of 300 and $\mathrm{AR}=4$; regions with near-zero velocity are highlighted. Figure 16 presents reattachment lines computed using the same procedure employed by Armaly et al., ${ }^{13}$ i.e., reattachment lines are defined as lines in which the streamwise velocity component is zero on a plane adjacent to the bottom wall $(0.0001 \mathrm{~m})$. The figure includes the reattachment lines, plotting the evolution of $x_{u}$ with the $z$-coordinate over half of the channel for AR $=4$ (Figure 16(a))

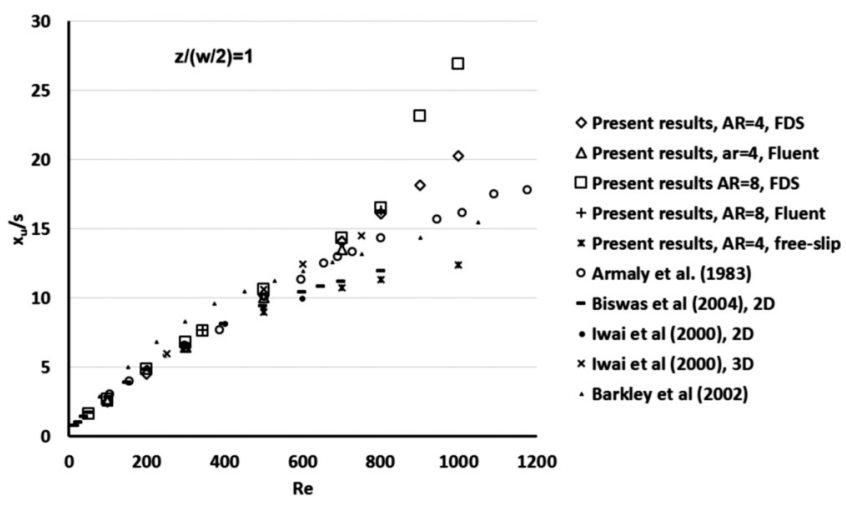

FIG. 14. Evolution of the reattachment length with Re in the different computations and compared with results reported in the literature. 

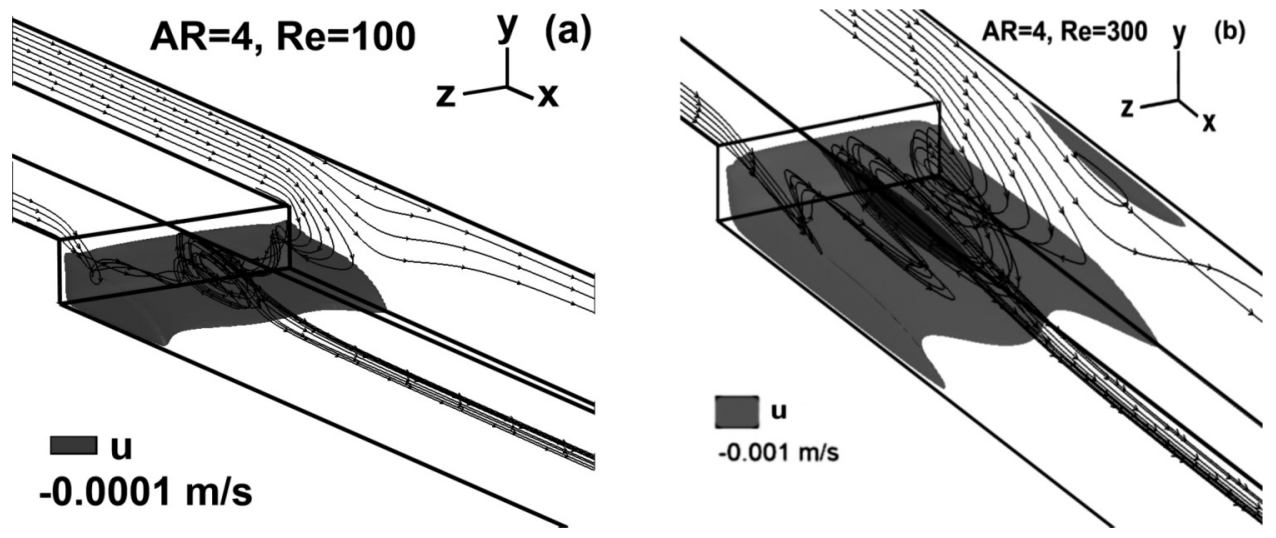

FIG. 15. Flow streamlines in the $\mathrm{AR}=4$ channel in the region of the step. Low velocity regions are highlighted. (a) shows the $\operatorname{Re}=100$ case, whereas the case of $\operatorname{Re}=300$ is shown in (b), in which the effect of the secondary bubble can be observed.

and $\mathrm{AR}=8$ (Figure 16(b)) for different Reynolds numbers. As the Reynolds increased, the secondary eddy became deeper, and its blockage effects in the zone next to the lateral walls were enhanced. Hence, the difference between the maximum and minimum of the reattachment line $\left(\mathrm{x}_{\mathrm{u}}\right)$ increased. Moreover, the absence of this blockage effect in the mid-plane region (because the secondary bubble was not present) and stronger wall-jets resulted in an increased length of the primary eddy in the mid-plane.

However, comparing both aspect ratios, the strength of the wall-jets was higher for Reynolds numbers greater than 800 , increasing the secondary eddy and its blockage effect in the AR $=8$ channel. The reattachment line was straightened about the symmetry plane and elongated in the streamwise direction. These findings agree with the results reported by Sheu and $\mathrm{Rani}^{22}$ and were likely due to distortions induced by a shedding-type instability, which did not appear for AR $=4$ at the same Reynolds numbers. All results presented were obtained for the same condition, when the solution was asymptotically steady. Reaching this condition required simulating approximately $10 \mathrm{~s}$ of physical time depending on the Reynolds number until the quasi-steady state was ensured. For cases with low Re, the state was steady; for the highest Re, an average over the last 100 time steps was used.

\section{B. Evolution of secondary eddy. Effect of the Reynolds number and aspect ratio}

In this section, the dependence of the main dimensions (defined in Figure 2) of the upper wall recirculation zone on the Reynolds number for $\mathrm{AR}=4$ and $\mathrm{AR}=8$ is discussed. The results presented in Figures 17-20 show the detachment and reattachment positions, x2 and x3, which
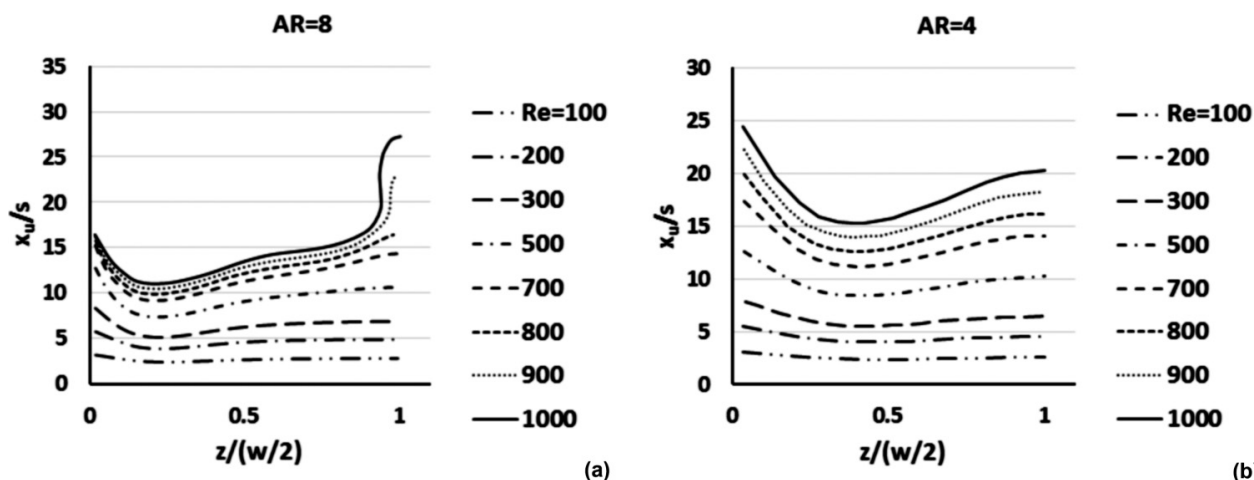

FIG. 16. Reattachment lines over half of the channel for $A R=8$ (a) and $A R=4$ (b) for different Reynolds numbers. 


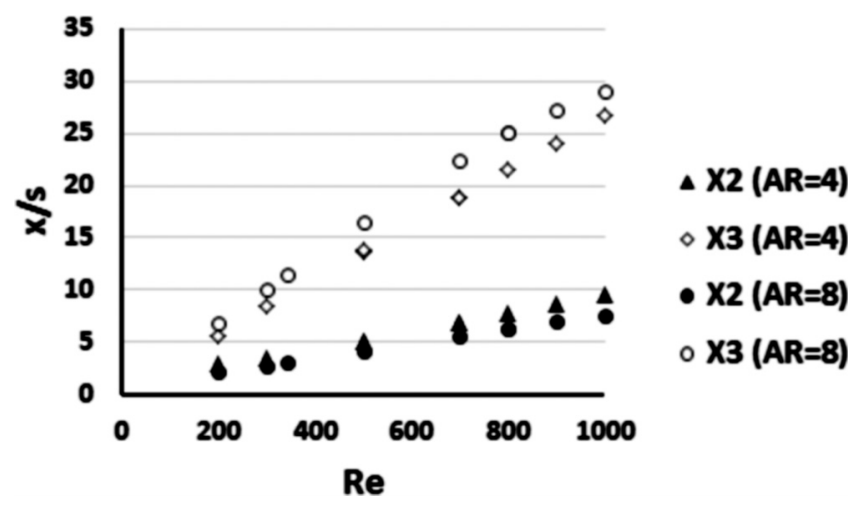

FIG. 17. Evolution of the distances to the detachment $(\mathrm{x} 2)$ and reattachment $(\mathrm{x} 3)$ points of the secondary bubble with the flow Reynolds number for the channels with $\mathrm{AR}=4$ and $\mathrm{AR}=8$.

indicate the length of the secondary eddy and its depth in spanwise (z) and vertical (y) directions from the lateral and top wall, respectively. All these distances and dimensions are represented in dimensionless form by dividing them by the step size, $h$, for the reattachment lengths and bubble depth, and half of the channel length for the spanwise dimension.

As the Reynolds number was increased, the secondary eddy moved downstream (x2 increases), increasing simultaneously in size in the streamwise direction $(\mathrm{x} 3-\mathrm{x} 2)$ and at the same rate for AR $=4$ and $\mathrm{AR}=8$. Figure 17 shows the increase in both reattachment lengths ( $\mathrm{x} 2$ and $\mathrm{x} 3$ ) with the Reynolds number. Figure 18 presents the size of the bubble in the streamwise direction. However, the increase in the dimensionless size in the spanwise direction was higher for $\mathrm{AR}=8$ than for $\mathrm{AR}$ $=4$, as shown in Figure 19, in which the spanwise variation of the secondary eddy is depicted for the investigated range of Reynolds numbers. The results for the depth along the vertical direction are analogous to those for the spanwise direction (Figure 20). Thus, for $\mathrm{AR}=8$ and Reynolds numbers greater than 800 , the secondary and the primary eddies coalesce in planes close to the sidewall, which did not occur for $\mathrm{AR}=4$, as shown in Figure 21. This finding reveals the importance of the aspect ratio.

\section{Numerical experiment: Free-slip boundary condition on lateral walls}

At low and moderate Reynolds numbers, the three-dimensional structure of the flow for low aspect ratios was due to the effect produced by the three-dimensional geometry of BFS and depended on the aspect ratio of the system. Thus, the results do not represent the intrinsic three-dimensional instability of the separate turbulent flow. However, it is well known that for

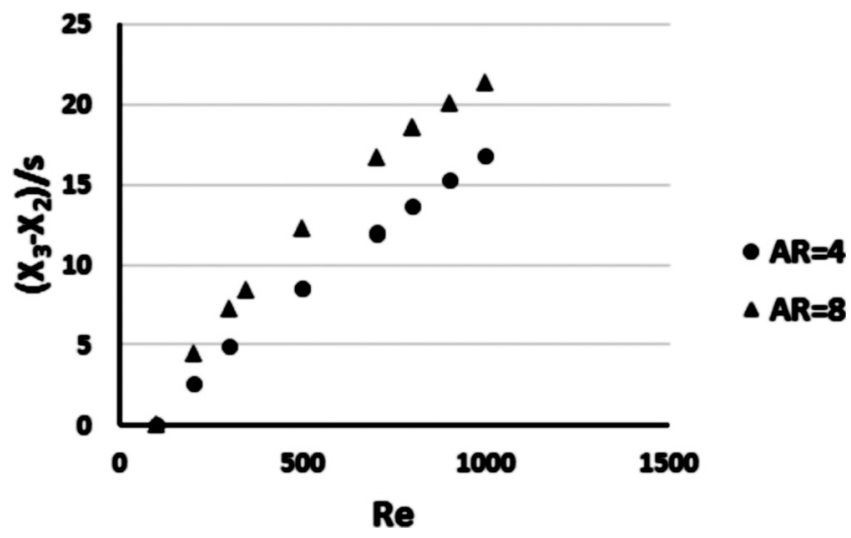

FIG. 18. Evolution of the streamwise length of the secondary bubble with the flow Reynolds number for both channels. 


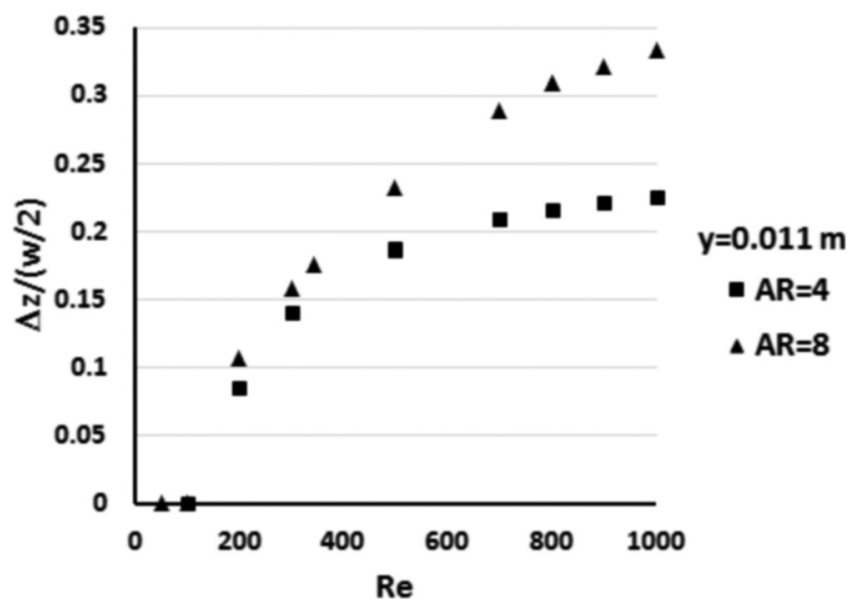

FIG. 19. Evolution of the spanwise length of the secondary bubble with the flow Reynolds number for both channels.

increasing Reynolds numbers in a BFS flow in the absence of sidewalls, the mean steady flow becomes three-dimensional due to three-dimensional instability.

To isolate the sidewall effects, numerical experiments were carried out using the same geometry, keeping the same expansion ratio and aspect ratio and sweeping the targeted range of Reynolds number but switching to free-slip boundary condition on the lateral walls. The computations were performed with the FDS code on the same grid used in the computations with the no-slip boundary condition on lateral walls.

The free-slip condition enforces a zero normal velocity and a zero streamwise velocity normal gradient at the walls. Hence, using the free-slip condition on both sidewalls is expected to result in a flow field, independent of the spanwise direction, where the reattachment point for each value of $\mathrm{z}$ is the same and the reattachment line is a straight line.

For detection and visualization of early three-dimensional flows, the vortex core lines were calculated with the $\lambda 2$ method described by Jeong and Hussain. ${ }^{40}$ Figure 22 (a) depicts the results obtained at Reynolds numbers ranging from 300 to 1000 for a channel with $\mathrm{AR}=4$. The results obtained are included in Figure 14 for completeness. As a first finding, the secondary separation zone appeared at a greater Reynolds number than for the no-slip boundary condition, and it moved downstream as the Reynolds number increased. Moreover, the flow field was z-independent until a Reynolds number of 1000 was reached. This finding is reflected in the reattachment length plotted in Figure 14. For Reynolds numbers less than 500, the reattachment length was the same for both cases, for the free-slip and no-slip boundary conditions. As the Reynolds reached 500, the reattachment length for the free-slip boundary condition cases became smaller than the reattachment length in the mid-plane for no-slip walls. This decrease occurred because of the blockage effect on the flow induced by the secondary eddy, which spread throughout the entire span of the channel.

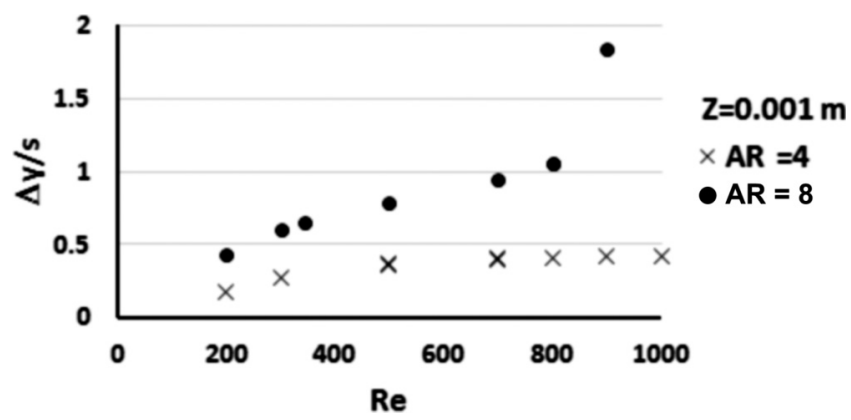

FIG. 20. Evolution of the bubble depth (vertical direction) with the flow Reynolds number for both channels. 

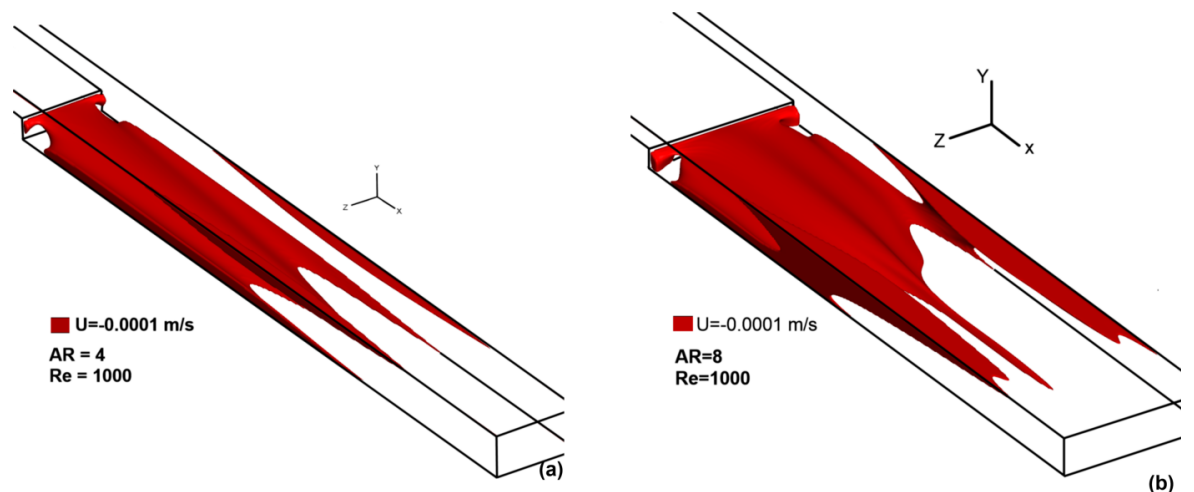

FIG. 21. Isosurfaces where the streamwise velocity changes sign for $\mathrm{Re}=1000$ and $\mathrm{AR}=4$ (a) and $\mathrm{AR}=8$ (b).

At a Reynolds number of 1000, the flow began to show three-dimensional structures even when the free-slip boundary condition was enforced on the sidewalls. These three-dimensional structures were clearly visible for a Reynolds number of 1200, as shown in Figure 22(b). In this case, the effect of the AR was further studied. The structures depicted in Figure 22(b) are similar to the typical vortical structures observed during transition in plane channel flow, in which the two-dimensional disturbances generated within the boundary layer rapidly evolve into a three-dimensional perturbation with a triangular shape ( $\Lambda$-vortices).

To discard the notion that the observed deviations were due to numerical noise caused by poor discretization resolution, an additional mesh size sensitivity study was conducted on a finer grid in
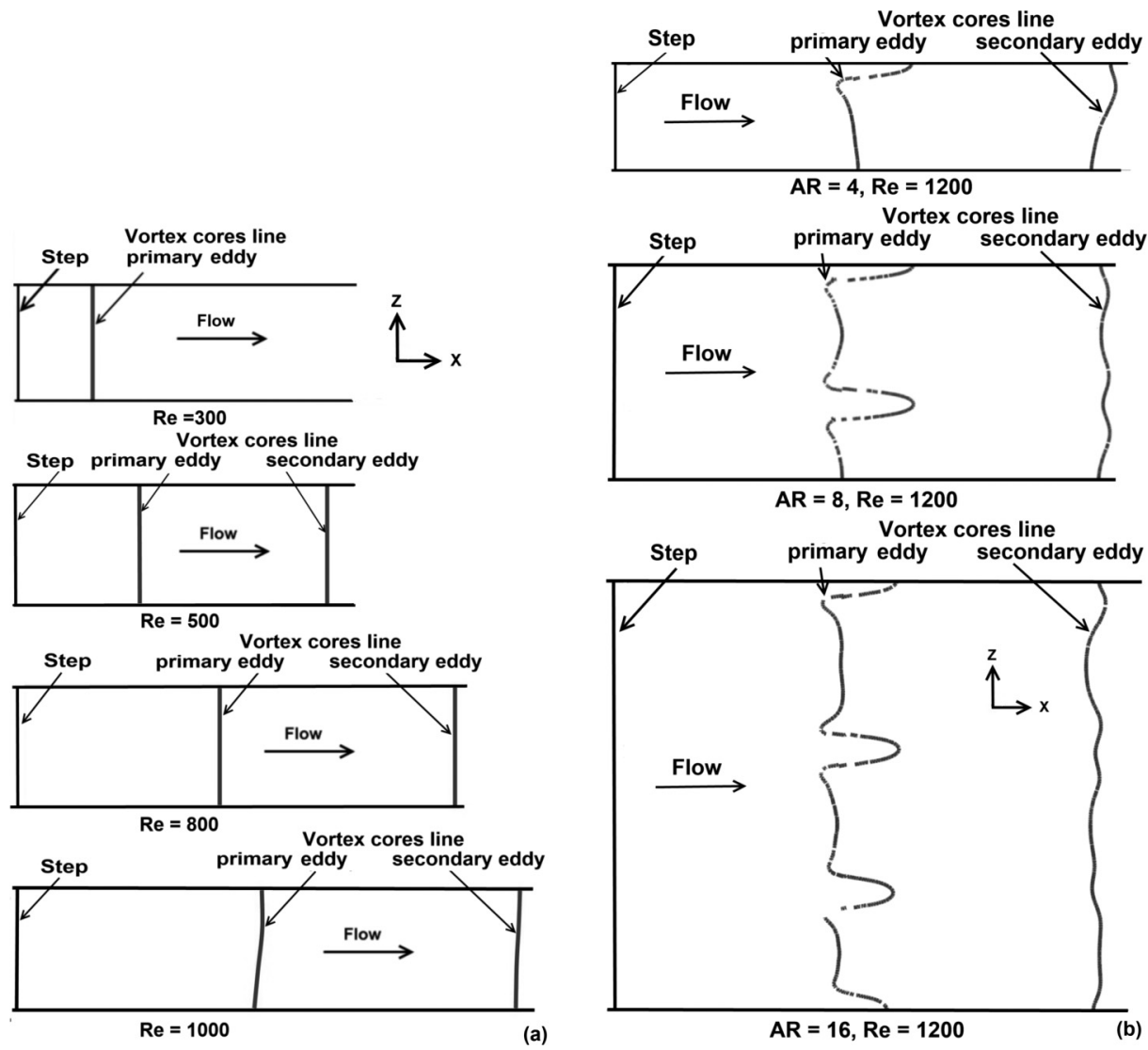

FIG. 22. Vortex core lines as defined by Jeong and Hussain. ${ }^{40}$ (a) Evolution of the primary and secondary vortex core lines with $\mathrm{Re}$ in the $\mathrm{AR}=4$ case. (b) Effect of the AR for $\mathrm{Re}=1200$. 
which the results were replicated, proving that the observed flow structures were independent of the mesh size used.

The results obtained demonstrate that for $\mathrm{ER}=2, \mathrm{AR}=4$, and the free-slip boundary condition, the flow structure becomes three-dimensional and steady at a Reynolds number of approximately 1000 , which is not due to the boundary layer of lateral walls. These results agree with those obtained by other authors, ${ }^{6}$ who have shown that, for ER $=2$ and in the absence of sidewalls, the flow structure becomes three-dimensional and steady at a Reynolds number of approximately 1000 because of a three-dimensional instability.

\section{CONCLUSIONS}

This paper presents a combined experimental and numerical study with the main objective of gaining a deeper understanding of the flow in a backward-facing step. In particular, the study focused on the appearance and the characteristics of the upper recirculation bubble and its effects on the flow. A good understanding of the flow behavior, particularly the size of the secondary bubble, will provide new opportunities for exerting flow control through boundary layer control.

An experimental apparatus reproducing the BFS geometry was developed for qualitative flow visualization. The effects of the flow Reynolds number and channel aspect ratio were analyzed by conducting several experiments. Horizontal planes at different heights showed that the upper recirculation zone adjacent to the sidewall was limited to a region lower than the step height, but the three-dimensional effects caused by the wall (wall-jets) extended through the entire span close to the bottom wall. The results obtained for the larger aspect ratio are analogous to those obtained for AR $=4$, but all the dimensions of the secondary bubble become larger.

Numerical simulations were performed with two objectives: to facilitate the interpretation of the flow visualizations and to gain a deeper understanding of the flow behavior. In this work, two different codes (FDS and Ansys-Fluent) were used to validate the results. Domain size and mesh-independence analyses were performed. Simulations were validated by comparison with experimental measurements reported in the literature. The agreement between the experimental flow visualization and numerically predicted stream traces was very good. The secondary bubble readily appeared at low Reynolds numbers $(\mathrm{Re}=200)$, and its length in the spanwise direction only covered approximately one-third to half of the step height. The secondary bubble grew in the spanwise direction with the Reynolds number and moved downstream from the step. For the smaller aspect ratio considered $(\mathrm{AR}=4)$, no coalescence of the primary or secondary bubble was observed over the range of Reynolds numbers studied. However, for the larger AR, this coalescence appeared close to the sidewall. How the sidewall effects distorted the flow adjacent to the lateral wall in planes next to the top wall was also observed. The results also confirm that the wall-jets were responsible for the minimum and maximum reattachment lengths near the lateral wall and the vanishing of the secondary bubble in the mid-plane.

The effects of the Reynolds number and aspect ratio on the secondary bubble dimensions were addressed as a function of the flow Reynolds number and channel aspect ratio using numerical solutions. The main conclusion is that as the Reynolds is increased, the secondary eddy moves downstream, growing simultaneously in size along the streamwise direction and at the same rate for $\mathrm{AR}=4$ and $\mathrm{AR}=8$. However, the increase in the dimensionless size along the spanwise and vertical directions is higher for $\mathrm{AR}=8$ than for $\mathrm{AR}=4$.

The effect of the secondary bubble on the primary reattachment length was studied. At the mid-plane, the reattachment length determined based on the three-dimensional CFD results matches the two-dimensional data derived from the literature below $\mathrm{Re}=400$. For higher Reynolds numbers, the results begin to deviate, and the three-dimensional length becomes higher than the corresponding two-dimensional one. This variation is caused by the existence of the upper wall recirculation zone adjacent to the sidewall, which enhances the existing wall-jets, causing the reattachment length at the mid-plane to increase. The interaction of the flow deviation (due to the upper eddy) and the sidewalls was observed to yield a swirling flow, which generated the maximum and minimum values of the reattachment length close to the sidewalls. As the Reynolds number was increased, 
the secondary eddy became deeper, and its blockage effect in the zone next to the lateral walls was enhanced. Hence, the difference between the maximum and minimum reattachment lengths increased as well. A comparison of the $\mathrm{AR}=8$ case with the $\mathrm{AR}=4$ one showed that the strength of the wall-jets increased with AR, thus increasing the blockage effect.

Finally, to decouple the sidewall effects due to the non-slip condition and the intrinsic flow three-dimensionality, numerical experiments with free-slip boundary conditions over the sidewalls were performed. In this case, the results were analyzed using the vortex core lines of the primary and secondary eddies. The first conclusion is that the secondary recirculation zone appears at a higher Reynolds number, but then occurs over the entire span, and that the solution does not depend on $\mathrm{z}$ for Reynolds numbers lower than 1000. In fact, the reattachment length is the same in both cases (no-slip and free-slip) up to $\operatorname{Re}=500$. At $\operatorname{Re}=1000$, the flow shows three-dimensional structures, typical for a transition in plane channel flows ( $\Lambda$-vortices). In summary, up to a Reynolds number of approximately 1000, the three-dimensionality is derived from the effect of the walls, whereas for higher Reynolds, three-dimensional instabilities inherent to the flow arise.

Interesting applications of the results obtained in the present study include the assessment of the effect of three-dimensional flow behaviors on heat transfer in narrow channels and the differences with respect to the classical 2D solutions, or the exertion of flow control via of the boundary layer. A deeper analysis of the flow structures and the effect of the pressure gradients formed in the channel on the changes in primary and secondary bubbles will be pursued by the authors in future investigations.

${ }^{1}$ J. K. Eaton and J. P. Johnston, “A review of research on subsonic turbulent flow reattachment,” AIAA J. 19(9), 1093-1100 (1981).

2 T. Lee and D. Mateescu, "Experimental and numerical investigation of 2-D backward-facing step flow," J. Fluids Struct. 12(6), 703-716 (1998).

${ }^{3}$ B. F. Armaly, F. Durst, J. C. F. Pereira, and B. Schönung, "Experimental and theoretical investigation of backward-facing step flow," J. Fluid Mech. 127, 473-496 (1983).

${ }^{4}$ G. Biswas, M. Breuer, and F. Durst, "Backward-facing step flows for various expansion ratios at low and moderate Reynolds numbers," J. Fluids Eng. 126(3), 362-374 (2004).

${ }^{5}$ L. Kaiktsis, G. Em Karniadakis, and S. A. Orszag, "Unsteadiness and convective instabilities in two-dimensional flow over a backward-facing step,” J. Fluid Mech. 321, 157-187 (1996).

${ }^{6}$ D. Barkley, M. G. M. Gomes, and R. D. Henderson, "Three-dimensional instability in flow over a backward-facing step," J. Fluid Mech. 473, 167-190 (2002).

${ }^{7}$ J. F. Beaudoin, O. Cadot, J. L. Aider, and J. E. Wesfreid, "Three-dimensional stationary flow over a backward-facing step," Eur J. Mech.-B/Fluids 23(1), 147-155 (2004).

${ }^{8}$ H. M. Blackburn, D. Barkley, and S. J. Sherwin, "Convective instability and transient growth in flow over a backward-facing step,” J. Fluid Mech. 603, 271-304 (2008).

${ }^{9}$ D. Lanzerstorfer and H. C. Kuhlmann, "Global stability of the two-dimensional flow over a backward-facing step," J. Fluid Mech. 693, 1-27 (2012).

${ }^{10}$ J. H. Nie and B. F. Armaly, "Three-dimensional convective flow adjacent to backward-facing step-effects of step height," Int. J. Heat Mass Transfer 45(12), 2431-2438 (2002).

${ }^{11}$ J. H. Nie and B. F. Armaly, "Reattachment of three-dimensional flow adjacent to backward-facing step," J. Heat Transfer 125(3), 422-428 (2003).

12 J. H. Nie and B. F. Armaly, "Reverse flow regions in three-dimensional backward-facing step flow," Int. J. Heat Mass Transfer 47(22), 4713-4720 (2004)

${ }^{13}$ B. F. Armaly, A. Li, and J. H. Nie, "Measurements in three-dimensional laminar separated flow," Int. J. Heat Mass Transfer 46(19), 3573-3582 (2003)

${ }^{14}$ M. S. Liou, L. A. Povinelli, and A. Arnone, Numerical Simulations of Three-Dimensional Laminar Flow Over a Backward Facing Step; Flow Near Side Walls (National Aeronautics and Space Administration, 1993), Vol. 106248.

${ }^{15}$ P. T. Williams and A. J. Baker, "Numerical simulations of laminar flow over a 3D backward-facing step," Int. J. Numer. Methods Fluids 24(11), 1159-1183 (1997).

${ }^{16}$ T. P. Chiang and T. W. Sheu, "A numerical revisit of backward-facing step flow problem," Phys. Fluids 11(4), 862-874 (1999).

${ }^{17}$ N. Tylli, L. Kaiktsis, and B. Ineichen, "Sidewall effects in flow over a backward-facing step: Experiments and numerical simulations," Phys. Fluids 14(11), 3835-3845 (2002).

${ }^{18}$ N. A. Malamataris, "A numerical investigation of wall effects in three-dimensional, laminar flow over a backward facing step with a constant aspect and expansion ratio," Int. J. Numer. Methods Fluids 71(9), 1073-1102 (2013).

${ }^{19}$ H. Iwai, K. Nakabe, and K. Suzuki, "Flow and heat transfer characteristics of backward-facing step laminar flow in a rectangular duct," Int. J. Heat Mass Transfer 43(3), 457-471 (2000).

${ }^{20}$ H. P. Rani and T. W. Sheu, "Nonlinear dynamics in a backward-facing step flow," Phys. Fluids 18(8), 084101 (2006).

${ }^{21}$ H. P. Rani, T. W. Sheu, and E. S. Tsai, "Eddy structures in a transitional backward-facing step flow," J. Fluid Mech. 588, 43-58 (2007). 
${ }^{22}$ T. W. Sheu and H. P. Rani, "Exploration of vortex dynamics for transitional flows in a three-dimensional backward-facing step channel," J. Fluid Mech. 550, 61-83 (2006).

${ }^{23}$ J. Tihon, V. Pěnkavová, J. Havlica, and M. Šimčík, "The transitional backward-facing step flow in a water channel with variable expansion geometry,” Exp. Therm. Fluid Sci. 40, 112-125 (2012).

${ }^{24}$ G. L. Juste and P. Fajardo, "Assessment of experimental optical techniques for characterizing heat transfer using numerical simulations," Eng. Appl. Comput. Fluid Mech. 9(1), 84-98 (2015).

${ }^{25}$ V. Uruba and P. Jonáš, "Flow over back-facing step in a narrow channel," PAAM 12, 501-502 (2012).

${ }^{26}$ W. M. Kays, M. E. Crawford, and B. Weigand, Convective Heat and Mass Transfer (Tata McGraw-Hill Education, 2012).

${ }^{27}$ A. O. Demuren and R. V. Wilson, "Estimating uncertainty in computations of two-dimensional separated flows," J. Fluids Eng. 116(2), 216-220 (1994).

${ }^{28}$ A. J. Torregrosa, P. Fajardo, A. Gil, and R. Navarro, "Development of non-reflecting boundary condition for application in 3D computational fluid dynamics codes,” Eng. Appl. Comput. Fluid Mech. 6(3), 447-460 (2012).

${ }^{29}$ J. H. Ferziger and M. Peric, Computational Methods for Fluid Dynamics (Springer Science \& Business Media, 2012).

${ }^{30}$ M. Lesieur, O. Métais, and P. Comte, Large-Eddy Simulations of Turbulence (Cambridge University Press, 2005).

${ }^{31}$ J. Smagorinsky, "General circulation experiments with the primitive equations. I. The basic experiment," Mon. Weather Rev. 91(3), 99-164 (1963).

${ }^{32}$ D. K. Lilly, On the Application of the Eddy Viscosity Concept in the Inertial Sub-Range of Turbulence (National Center for Atmospheric Research, 1966).

${ }^{33}$ Y. Zang, R. L. Street, and J. R. Koseff, "A dynamic mixed subgrid-scale model and its application to turbulent recirculating flows," Phys. Fluids A 5(12), 3186-3196 (1993).

${ }^{34}$ M. Germano, U. Piomelli, P. Moin, and W. H. Cabot, "A dynamic subgrid-scale eddy viscosity model," Phys. Fluids A 3(7), 1760-1765 (1991).

${ }^{35}$ K. McGrattan, S. Hostikka, J. Floyd, G. Forney, T. Korhonen, and R. McDermott, Fire Dynamics Simulator (Version 6), Technical Reference Guide, NIST Special Publication Vol. 1018 (NIST, 2012).

${ }^{36}$ Ansys, Ansys Fluent, 14.0 Theory Guide, ANSYS, Inc., 2011.

${ }^{37}$ R. I. Issa, A. D. Gosman, and A. P. Watkins, "The computation of compressible and incompressible recirculating flows by a non-iterative implicit scheme," J. Comput.Phys. 62(1), 66-82 (1986).

${ }^{38}$ S. E. Kim, "Large eddy simulation using unstructured meshes and dynamic subgrid-scale turbulence models," AIAA Paper 2004-2548, 2004

${ }^{39}$ Y. Wang, K. Zhong, N. Zhang, and Y. Kang, "Numerical analysis of solar radiation effects on flow patterns in street canyons," Eng. Appl. Comput. Fluid Mech. 8(2), 252-262 (2014).

40 J. Jeong and F. Hussain, “On the identification of a vortex,” J. Fluid Mech. 285, 69-94 (1995). 